Article

\title{
Bright-Dark and Multi Solitons Solutions of (3 + 1)-Dimensional Cubic-Quintic Complex Ginzburg-Landau Dynamical Equation with Applications and Stability
}

\author{
Chen Yue, Dianchen Lu*(D), Muhammad Arshad *(D), Naila Nasreen and Xiaoyong Qian \\ Faculty of Science, Jiangsu University, Zhenjiang 212013, China; ychen108@uncc.edu (C.Y.); \\ naila_nasreen@hotmail.co.uk (N.N.); 5853868@163.com (X.Q.) \\ * Correspondence: dclu@ujs.edu.cn (D.L.); marshad@ujs.edu.cn (M.A.)
}

Received: 18 January 2020; Accepted: 2 February 2020; Published: 10 February 2020

check for updates

\begin{abstract}
In this paper, bright-dark, multi solitons, and other solutions of a $(3+1)$-dimensional cubic-quintic complex Ginzburg-Landau (CQCGL) dynamical equation are constructed via employing three proposed mathematical techniques. The propagation of ultrashort optical solitons in optical fiber is modeled by this equation. The complex Ginzburg-Landau equation with broken phase symmetry has strict positive space-time entropy for an open set of parameter values. The exact wave results in the forms of dark-bright solitons, breather-type solitons, multi solitons interaction, kink and anti-kink waves, solitary waves, periodic and trigonometric function solutions are achieved. These exact solutions have key applications in engineering and applied physics. The wave solutions that are constructed from existing techniques and novel structures of solitons can be obtained by giving the special values to parameters involved in these methods. The stability of this model is examined by employing the modulation instability analysis which confirms that the model is stable. The movements of some results are depicted graphically, which are constructive to researchers for understanding the complex phenomena of this model.
\end{abstract}

Keywords: modified extended simple equation and $\exp (-\phi(\xi))$-expansion methods; proposed F-expansion method; cubic-quintic complex Ginzburg-Landau equation; multi solitons; periodic solutions; solitary wave solutions

\section{Introduction}

The nonlinear Schrödinger's equations (NLSEs) are well-known models in nonlinear partial differential equations (PDEs) to govern the optical soliton propagation [1-13]. Since the 19th century, many researchers have focused on the PDEs with complex variables [14]. Succi was the pioneer who proposed the complex distribution of the Gross-Pitaevski model through utilizing a lattice Boltzmann model for Bose-Einstein condensation in 1998. After that, expanding this model to a higher-dimensional equation was described [15,16]. The authors in [17] proposed one more complex division for NLSE wherein the function of the wave is separated into phase angle and amplitude.

From NLSEs, the cubic-quintic complex Ginzburg-Landau model is a continuing estimate to the dynamics of the area in a passively mode-locked laser. It is admitted that this model is constructive in demonstrating imperative phenomena-for example, erbium-doped fiber amplifiers and propagation of ultra-short pulses in optical transmission lines having spectral filtering. In physics, the generalized quintic complex Ginzburg-Landau equation (GQCGLE) was utilized fruitfully in the formation of other non-equilibrium procedures. In the model, the quintic term explains the physical significance that is absent in existing models in a previous study [18]. It occurs in various 
branches of science and plays an important role in practical applications including fluid dynamics, nonlinear optics, mathematical biology, hydro dynamical stability problems, condensed matter physics, Bose-Einstein condensates, chemical reactions, super conductivity, and quantum field theories [19-23]. It demonstrates prosperous dynamics and has turned into an example for alterations to the chaos of spatio-temporal. In Refs. [24,25], some other aspects regarding the various forms of Ginzburg-Landau models are described. This model as well occurs in the investigation of chemical systems studied via reaction-diffusion equations. The GQCGLE Model also plays the part of a simplified form in fluid dynamic equations. Recently, numerous researchers have concentrated on this model that involve localized complex forms in optical media-for example, necklace-ring solitons, vortex solitons, and so on [26-28].

Complex Ginzburg-Landau models of higher dimensions from complex lattice Boltzmann models were proposed by the authors [29-31]. These problems were built on the complex lattice Boltzmann equations. In physics and applied mathematics, the different forms of complex Ginzburg-Landau equations have attained great attention from researchers, as universal models where the most interesting solutions are dissipative solitons. In optical lattices of nonlinear self-localized structures, one- and two-dimensional arrays frequently assigned as discrete solitons have been forecasted and examined [32]. In [20], mathematical and physical features of the equations based on GQCGLE were reviewed. As numerical tools, the lattice Boltzmann method (LBM) has been broadly used in several study areas for examining fluid dynamics in the previous decade. The key theme behind using a lattice Boltzmann technique is to implement a straightforward mesoscopic equation of a fluid flow, generally concerning some distinct particle velocities that are not enough for describing properly the macroscopic flow pattern as the macroscopic PDEs are improved from the mesoscopic equation preserving preferred physical quantities and the principal to accurate fluxes of the preserved quantities [33-35]. Different self-interaction potentials were engineered in order to obtain nontrivial analytic results, with the purpose of testing the robustness of regarding the soliton as the ground state of the hairy sector, and its key role in the microscopic counting of hairy black hole entropy [36].

In recent years, NLSEs have attracted much attention from the constructing solitons and numerical solutions due to them being widely used to explain nonlinear complex phenomena. Several conventional techniques are extracted to obtain exact solutions such as the Tanh and Sech methods, the inverse scattering method, the extended tanh method, the Hirota bilinear and Darbox transform methods, the Bäcklund transform method, the generalized F-expansion technique, the Jacobi elliptic function expansion technique, the reduced differential transform method, the modified direct algebraic technique, variational iteration methods, and several others [28-35,37-41]. The authors in [40] have been working on CGLEs using different techniques like the modified simple equation method. Inspired from the above works, we intend to construct a new soliton solution of the CQCGLE model using a modified extended simple equation method. The soliton profile is sensitive to entropy, i.e., due to the changes in the entropy amplitude and the width of solitons. It is also observed that the increasing ion temperature and increasing magnetic field affect the shape of the soliton.

In the current paper, the aim is to construct the novel analytical solutions in different forms such as dark-bright solitons, multi solitons, kink and anti-kink waves, solitary wave, and trigonometric function solutions of $(3+1)$-dimensional CQCGLE via the modified extended simple equation method (MESEM), $\exp (-\phi(\xi))$-expansion method and proposed F-Expansion method. The achieved solutions are exact and more general. The modulational instability (MI) analysis is discussed by employing standard stability analysis that confirms that the model is stable and the achieved solutions are also stable.

This paper is organized as follows. An introduction is given in Section 1. The mathematical model is described in Section 2. The important steps of the projected techniques are explained in Section 3. Section 4 applies the proposed methods on $(3+1)$-dimensional CQCGLE. In Section 5, the stability of this model is discussed by using modulation instability analysis. The discussion of results and their Physical Interpretations are explained in Section 6. The conclusions are revealed in Section 7. 


\section{Mathematical Model}

The (3 + 1)-dimensional CQCGLE [42] has the form of

$$
i \frac{\partial q}{\partial z}+\left(\frac{1}{2}-i \eta\right)\left(\frac{\partial^{2} q}{\partial x^{2}}+\frac{\partial^{2} q}{\partial y^{2}}+\frac{\partial^{2} q}{\partial t^{2}}\right)+i \gamma q+(1-i \lambda)|q|^{2} q-(v-i \mu)|q|^{4} q=0 .
$$

This model involves the derivative $\frac{\partial}{\partial z}$ which is spatial, and the system evolution depends on the $z$ coordinate. The $\lambda$ is the coefficient that accounts for the linear gain $(\lambda>0)$ or loss $(\lambda<0), \eta$ shows effective diffusion, $\lambda$ is used here for the cubic gain coefficient, and $\mu$ accounts for the coefficient of quintic-loss. All parameters in this model are positive for knowing the physical meaning of these coefficients. In Equation (1), the term $\gamma q+(1-i \lambda)|q|^{2} Q-(v-i \mu)|q|^{4} q$ can be observed as source term.

\section{Elucidation of Proposed Methods}

In this portion, we will explain the algorithm of MESEM and the exp $(-\phi(\xi))$-expansion scheme for seeking the solitons solutions of nonlinear PDEs in this section. We suppose a common nonlinear PDE, say in four independent variables $x, y, z$ and $t$, is

$$
F\left(q, q_{t}, q_{x}, q_{y}, q_{z}, q_{t t}, q_{x x}, q_{y y}, q_{z z}, \ldots\right)=0,
$$

where $F$ is a polynomial function with respect to some specific variables that involve nonlinear and linear terms of advanced order derivatives, and the $q(x, y, z, t)$ is an unknown function. By utilizing transformation, the $q(x, y, z, t)$ can be converted to a polynomial function through which the complex variable can be combined into real variables.

Consider the transformation for reducing independent variables into a unique variable as

$$
q(x, y, z, t)=\psi(\xi), \quad \xi=k_{1} x+k_{2} y+k_{3} z+\omega t,
$$

where $k_{1}, k_{2}, k_{3}$ are different wavelengths and $\omega$ shows frequency. Through employing transformation (3), Equation (2) is transformed into ODE as

$$
G\left(\psi, \psi^{\prime}, \psi^{\prime \prime}, \psi^{\prime \prime \prime}, \ldots\right)=0,
$$

where $G$ is a polynomial and " $(')$ " indicates derivative of $\psi$ with respect to $\xi$.

\subsection{Modified Extended Simple Equation Technique}

The main steps of proposed technique are:

Step 1: We suppose the solution of Equation (4) has the form of:

$$
\psi(\xi)=\sum_{i=0}^{N} A_{i}\left(\frac{\phi^{\prime}}{\phi}\right)^{i}+\sum_{j=0}^{N-1} B_{j}\left(\frac{\phi^{\prime}}{\phi}\right)^{j}\left(\frac{1}{\phi}\right),
$$

where $A_{i}$ and $B_{j}$ are real constants to be determined later. In addition, $\phi(\xi)$ satisfies the below second order equation

$$
\phi^{\prime \prime}(\xi)+\Omega \phi(\xi)=\rho,
$$


where $\Omega$ and $\rho$ are arbitrary constants. Equation (6) gives three kinds of general solutions having two arbitrary parameters as follows:

$$
\begin{gathered}
\phi(\xi)=\left\{\begin{array}{l}
A_{1} \cosh (\sqrt{-\Omega} \xi)+A_{2} \sinh (\sqrt{-\Omega} \xi)+\frac{\rho}{\Omega}, \quad \Omega<0, \\
A_{1} \cosh (\sqrt{\Omega} \xi)+A_{2} \sinh (\sqrt{\Omega} \xi)+\frac{\rho}{\Omega}, \quad \Omega>0, \\
\frac{\mu}{2} \xi^{2}+A_{1} \xi+A_{2}, \quad \Omega=0 .
\end{array}\right. \\
\left(\frac{\phi^{\prime}}{\phi}\right)^{2}=\left\{\begin{array}{l}
\left(\Omega A_{1}^{2}-\Omega A_{2}^{2}-\frac{\rho^{2}}{\Omega}\right)\left(\frac{1}{\phi}\right)^{2}-\Omega+\frac{2 \rho}{\Omega}, \quad \Omega<0, \\
\left(\Omega A_{1}^{2}+\Omega A_{2}^{2}-\frac{\rho^{2}}{\Omega}\right)\left(\frac{1}{\phi}\right)^{2}-\Omega+\frac{2 \rho}{\Omega}, \quad \Omega>, 0 \\
\left(A_{1}^{2}-2 \Omega A_{2}\right)\left(\frac{1}{\phi}\right)^{2}+\frac{2 \rho}{\Omega}, \quad \Omega=0,
\end{array}\right.
\end{gathered}
$$

where the constants $A_{1}$ and $A_{2}$ are arbitrary.

Step 2: $N$ is determined via utilizing the balancing principle among the terms of highest order derivative and the nonlinear in Equation (4), and is positive.

Step 3: Putting Equation (5) along with Equation (6) into Equation (4) and equating the coefficients of different powers of $\frac{1}{\phi^{\iota}(\xi)}$ and $\left(\frac{\phi^{\prime}(\xi)}{\phi(\xi)}\right)\left(\frac{1}{\phi^{\prime}(\xi)}\right)$ to zero capitulates a set of equations. By using Mathematica software, these sets of equations are resolved, and then the value of parameters are obtained.

Step 4: Substituting the values of parameters obtained in Step 3 and general solutions (7) of Equation (6) into Equation (5), solutions of Equation (3) can then be obtained in a concise way.

\section{2. $\operatorname{Exp}(-\phi(\xi))$-Expansion Method}

The main steps of this technique are:

Step 1: We suppose the solution of Equation (4) has the form:

$$
\psi(\xi)=\sum_{i=-N}^{N} B_{-i}(\exp (-\phi(x)))^{i}
$$

where $B_{i}$ are real constants to be determined later. In addition, $\phi(\xi)$ satisfies the below second order equation

$$
\phi^{\prime}(\xi)=\exp (-\phi(x))+a \exp (\phi(x))+b
$$

where $a$ and $b$ are arbitrary constants. Equation (6) gives five kinds of general solutions as

Type I When $a \neq 0, b^{2}-4 a>0$,

$$
\phi(\xi)=\ln \left(-\frac{\sqrt{b^{2}-4 a} \tanh \left(\frac{\sqrt{b^{2}-4 a}}{2}(\xi+c)\right)+b}{2 a}\right) .
$$

Type II When $a \neq 0, b^{2}-4 a<0$,

$$
\phi(\xi)=\ln \left(\frac{\sqrt{4 a-b^{2}} \tan \left(\frac{\sqrt{4 a-b^{2}}}{2}(\xi+c)\right)-b}{2 a}\right) .
$$

Type III When $a=0, b \neq 0 \& b^{2}-4 a>0$,

$$
\phi(\xi)=-\ln \left(\frac{b}{\exp (b(\xi+c))-1}\right) .
$$


Type IV When $a \neq, b \neq 0 \& b^{2}-4 a=0$,

$$
\phi(\xi)=\ln \left(-\frac{2 b(\xi+c)+2}{b^{2}(\xi+c)}\right) .
$$

Type V When $a=0,=0 \& b^{2}-4 a=0$,

$$
\phi(\xi)=\ln (\xi+c)
$$

Step 2: $N$ is determined via using the balancing principle among the terms of highest order derivative and the nonlinear in Equation (4), and is positive.

Step 3: Substituting Equation (9) along with Equation (10) into Equation (4) and equating the coefficients of different powers of $(\exp (-\phi(x)))^{i}$ to zero capitulates a set of equations. By using Mathematica software, these sets of equations are resolved, and then the value of parameters are obtained.

Step 4: Substituting the values of parameters obtained in Step 3 and general solutions (11) to (15) of Equation (10) into Equation (9), solutions of Equation (3) can be then be obtained in a concise way.

\subsection{Proposed F-Expansion Method}

The main steps of proposed technique are:

Step 1: We suppose the solution of Equation (4) has the form:

$$
\psi(\xi)=\sum_{i=0}^{N} A_{i}(a+F(\xi))^{i}+\sum_{j=-1}^{-N} B_{-j}(a+F(\xi))^{j},
$$

where $A_{i}, B_{-j}$ and $a$ are real constants. In addition, $F(\xi)$ satisfies the ODE

$$
F^{\prime}(\xi)=d_{0}+d_{1} F(\xi)+d_{2} F^{2}(\xi)+d_{3} F^{3}(\xi),
$$

where $d_{i} i=0,1,2,3$ are arbitrary constants.

Step 2: $N$ is a positive integer which is determined via utilizing the balancing principle among the terms of highest order derivative and the nonlinear in Equation (4).

Step 3: Putting Equations (16) along with (17) into (4) and equating the coefficients of different powers of $\frac{F^{j}(\xi)}{(a+F(\xi))^{k}}$ to zero capitulates a set of equations. By using Mathematica software, these sets of equations are resolved, and then the value of parameters are obtained.

Step 4: Substituting the values of parameters obtained in the previous step and solutions of Equation (17) into Equation (16), solutions of Equation (3) can then be obtained.

\section{Solitons Solutions of $(3+1)$-Dimensional Cubic-Quintic Complex Ginzburg-Landau Equation}

In this part, we will apply modified extended SEM and $\exp (-\phi(\xi))$-expansion scheme to get a soliton solution. As the Equation (1) is complex, assume the solution in a traveling wave form as follows:

$$
q(x, y, z, t)=\psi(\xi) e^{i P}, \quad P=\alpha_{1} x+\alpha_{2} y+\alpha_{3} z+\tau t+\epsilon .
$$

Substitute Equation (18) into Equation (1) and make separate imaginary and real parts as

$$
\begin{gathered}
\left(\tau^{2}+\alpha_{1}^{2}+\alpha_{2}^{2}+2 \alpha_{3}\right) \psi-2 \psi^{3}+2 \nu \psi^{5}-4 \eta\left[\left(\tau \omega+k_{1} \alpha_{1}+k_{2} \alpha_{2}\right) \psi^{\prime}-\left(\omega^{2}+k_{1}^{2}+k_{2}^{2}\right) \psi^{\prime \prime}=0 .\right. \\
\left(-2 \gamma-2 \eta \tau^{2}-2 \eta \alpha_{1}^{2}\right) \psi-2 \eta \alpha_{2}^{2}+2 \lambda \psi^{3}-2 \mu \psi^{5}-\left(2 \tau \omega+2 k_{3}+2 k_{1} \alpha_{1}+2 k_{2} \alpha_{2}\right) \psi^{\prime}+2 \eta\left(\omega^{2}+k_{1}^{2}+k_{2}^{2}\right) \psi^{\prime \prime}=0
\end{gathered}
$$


Using Equation (20) in Equation (19), we have

$$
\begin{aligned}
& \left(\left(\tau^{2}+\alpha_{1}^{2}+\alpha_{2}^{2}+2 \alpha_{3}\right)-4 v\left(\gamma+\eta \tau^{2}+\eta \alpha_{2}^{2}\right)\right) \psi-4 \eta\left(\left(\tau \omega+k_{1} \alpha_{1}+k_{2} \alpha_{2}\right)+\left(\tau \omega+2 k_{3}+2 k_{1} \alpha_{1}+2 k_{2} \alpha_{2}\right)\right) \psi^{\prime} . \\
& -\left(\left(\omega^{2}+k_{1}^{2}+k_{2}^{2}\right)-4 v \eta\left(\omega^{2}+k_{1}^{2}+k_{2}^{2}\right)\right) \psi^{\prime \prime}-(2+4 v \lambda) \psi^{3}=0 .
\end{aligned}
$$

\subsection{Solitons Solutions by Extended SEM}

In this sub-part, extended SEM is employed to construct the solution of Equation (1). Apply a homogeneous balance principle on Equation (21) and assume the solution as

$$
\psi(\xi)=A_{0}+A_{1} \frac{\phi^{\prime}(\xi)}{\phi(\xi)}+B_{0} \frac{1}{\phi(\xi)} .
$$

Substituting Equation (22) along Equation (6) in Equation (21) and setting the coefficients to zero of $\left(\frac{\phi^{\prime}(\xi)}{\phi(\xi)}\right)^{j} \frac{1}{\phi^{k}(\xi)}$, we obtain a set of equations in parameters $B_{0}, A_{0}, A_{1}, \lambda, \eta$, and $v$. This algebraic set of equations is solved by utilizing Mathematica 9 . The following cases are achieved as

Case 1: If $\Omega>0$,

Set 1:

$$
\begin{aligned}
& A_{0}=B_{0}=0, k_{2}= \pm \sqrt{-k_{1}^{2}-\omega^{2}}, k_{3}=\frac{3 \alpha_{2} \sqrt{-k_{1}^{2}-\omega^{2}}-\alpha_{1} k_{1}-2 \tau \omega}{2}, a_{2}=-\frac{\sqrt{\rho^{2}-a_{1}^{2} \Omega^{2}}}{\Omega}, \\
& \alpha_{3}=\frac{\Omega\left(\alpha_{2}^{2}(4 \eta v-1)-\alpha_{1}^{2}+4 \gamma v+4 \eta v \tau^{2}-\tau^{2}\right)+2 A_{1}^{2}(2 \lambda \nu+1)\left(2 \rho-\Omega^{2}\right)}{2 \Omega} .
\end{aligned}
$$

Set 2:

$$
\begin{gathered}
A_{0}= \pm \frac{A_{1} \sqrt{2 \rho-\Omega^{2}}}{\sqrt{\Omega}}, k_{2}=-\sqrt{-k_{1}^{2}-\omega^{2}}, k_{3}=\frac{3 \alpha_{2} \sqrt{-k_{1}^{2}-\omega^{2}}-\alpha_{1} k_{1}-2 \tau \omega}{2}, a_{2}=-\frac{\sqrt{\rho^{2}-a_{1}^{2} \Omega^{2}}}{\Omega}, \\
B_{0}=0, \alpha_{3}=\frac{\Omega\left(\alpha_{2}^{2}(4 \eta v-1)-\alpha_{1}^{2}+4 \gamma v+4 \eta v \tau^{2}-\tau^{2}\right)+8 A_{1}^{2}(2 \lambda v+1)\left(2 \rho-\Omega^{2}\right)}{2 \Omega} .
\end{gathered}
$$

Set 3:

$$
\begin{aligned}
& A_{0}=\frac{3 B_{0}\left(\frac{\Omega^{2}}{\rho}-4\right)}{2 \Omega}, A_{1}=0, \alpha_{3}=\frac{2 \rho^{2} \Omega^{2}\left(\alpha_{2}^{2}(4 \eta v-1)-\alpha_{1}^{2}+4 \gamma v+\tau^{2}(4 \eta v-1)\right)+9 B_{0}^{2}(2 \lambda v+1)\left(\Omega^{2}-4 \rho\right)^{2}}{4 \rho^{2} \Omega^{2}} \\
& k_{2}= \pm \sqrt{\frac{9 B_{0}^{2}(2 \lambda v+1)\left(\Omega^{2}-4 \rho\right)+\rho^{2} \Omega(1-4 \eta v)\left(k_{1}^{2}+\omega^{2}\right)}{\rho^{2}(\Omega-4 \eta v \Omega)}}, a_{2}=-\frac{\sqrt{4 \rho^{2}\left(2 \Omega^{2}-9 \rho\right)-9 a_{1}^{2} \Omega^{2}\left(\Omega^{2}-4 \rho\right)}}{3 \sqrt{\Omega^{4}-4 \rho \Omega^{2}}} \\
& k_{3}= \pm \frac{3 \alpha_{2} \sqrt{\Omega\left(\rho^{2} \Omega(4 \eta v-1)\left(k_{1}^{2}+\omega^{2}\right)-9 B_{0}^{2}(2 \lambda v+1)\left(4 \rho-\Omega^{2}\right)\right)}}{2 \sqrt{\rho^{2} \Omega^{2}(1-4 \eta v)}}-\alpha_{1} k_{1}-2 \tau \omega .
\end{aligned}
$$

Exact Solutions of Equation (1) from solutions Sets (23), (24) and (25) are constructed as

$$
\begin{gathered}
q_{1}=-\frac{A_{1}\left(\sqrt{-\Omega} \sqrt{\rho^{2}-a_{1}^{2} \Omega^{2}} \cos (\xi \sqrt{-\Omega})+a_{1} \Omega^{3 / 2} \sin (\xi \sqrt{\Omega})\right)}{-\sqrt{\rho^{2}-a_{1}^{2} \Omega^{2}} \sin (\xi \sqrt{-\Omega})+a_{1} \Omega \cos (\xi \sqrt{\Omega})+\rho} e^{i P} . \\
q_{2}=\frac{A_{1}}{\sqrt{\Omega}}\left( \pm \sqrt{2 \rho-\Omega^{2}}-\frac{\lambda \Omega\left(\sqrt{\rho^{2}-a_{1}^{2} \Omega^{2}} \cos (\xi \sqrt{\Omega})+a_{1} \Omega \sin (\xi \sqrt{\Omega})\right)}{-\lambda \sqrt{\rho^{2}-a_{1}^{2} \Omega^{2}} \sin (\xi \sqrt{\Omega})+a_{1} \lambda \Omega \cos (\xi \sqrt{\Omega})+\rho \Omega}\right) e^{i P} . \\
q_{3}=B_{0}\left(\Omega\left(\frac{1}{a_{2} \Omega \sin (\xi \sqrt{\Omega})+a_{1} \Omega \cos (\xi \sqrt{\Omega})+\rho}+\frac{3}{2 \rho}\right)-\frac{6}{\Omega}\right) e^{i P} .
\end{gathered}
$$

Case 2: If $\Omega<0$, 
Set 1:

$$
\begin{aligned}
& A_{0}=B_{0}=0, k_{2}=-\sqrt{-k_{1}^{2}-\omega^{2}}, k_{3}=\frac{3 \alpha_{2} \sqrt{-k_{1}^{2}-\omega^{2}}-\alpha_{1} k_{1}-2 \tau \omega}{2}, a_{2}= \pm \frac{\sqrt{a_{1}^{2} \Omega^{2}-\rho^{2}}}{\Omega}, \\
& \alpha_{3}=\frac{\Omega\left(\alpha_{2}^{2}(4 \eta v-1)-\alpha_{1}^{2}+4 \gamma v+4 \eta v \tau^{2}-\tau^{2}\right)+2 A_{1}^{2}(2 \lambda \nu+1)\left(2 \rho-\Omega^{2}\right)}{2 \Omega} .
\end{aligned}
$$

Set 2:

$$
\begin{aligned}
& A_{0}= \pm \frac{A_{1} \sqrt{2 \rho-\Omega^{2}}}{\sqrt{\Omega}}, k_{2}=-\sqrt{-k_{1}^{2}-\omega^{2}}, k_{3}=\frac{3 \alpha_{2} \sqrt{-k_{1}^{2}-\omega^{2}}-\alpha_{1} k_{1}-2 \tau \omega}{2}, a_{2}=-\frac{\sqrt{a_{1}^{2} \Omega^{2}-\rho^{2}}}{\Omega}, \\
& B_{0}=0, \alpha_{3}=\frac{\Omega\left(\alpha_{2}^{2}(4 \eta v-1)-\alpha_{1}^{2}+4 \gamma v+4 \eta v \tau^{2}-\tau^{2}\right)+8 A_{1}^{2}(2 \lambda v+1)\left(2 \rho-\Omega^{2}\right)}{2 \Omega} .
\end{aligned}
$$

Set 3:

$$
\begin{aligned}
& A_{0}=\frac{3 B_{0}\left(\frac{\Omega^{2}}{\rho}-4\right)}{2 \Omega}, A_{1}=0, \alpha_{3}=\frac{2 \rho^{2} \Omega^{2}\left(\alpha_{2}^{2}(4 \eta v-1)-\alpha_{1}^{2}+4 \gamma v+4 \eta v \tau^{2}-\tau^{2}\right)+9 B_{0}^{2}(2 \lambda \nu+1)\left(\Omega^{2}-4 \rho\right)^{2}}{4 \rho^{2} \Omega^{2}}, \\
& k_{2}=\mp \sqrt{\frac{\Omega\left(\rho^{2} \Omega(4 \eta v-1)\left(k_{1}^{2}+\omega^{2}\right)-9 B_{0}^{2}(2 \lambda v+1)\left(4 \rho-\Omega^{2}\right)\right)}{\rho^{2} \Omega^{2}(1-4 \eta v)}}, a_{2}=-\frac{\sqrt{9 a_{1}^{2}\left(4 \rho \Omega^{2}-\Omega^{4}\right)+4 \rho^{2}\left(2 \Omega^{2}-9 \rho\right)}}{3 \sqrt{4 \rho \Omega^{2}-\Omega^{4}}}, \\
& k_{3}=\mp \frac{3 \alpha_{2} \sqrt{\rho^{2} \Omega(4 \eta v-1)\left(k_{1}^{2}+\omega^{2}\right)-9 B_{0}^{2}(2 \lambda v+1)\left(4 \rho-\Omega^{2}\right)}}{2 \sqrt{\rho^{2}(1-4 \eta v)}}-\alpha_{1} k_{1}-2 \tau \omega .
\end{aligned}
$$

Solitons solutions of Equation (1) from solutions (29), (30), and (31) as:

$$
\begin{gathered}
q_{4}=\frac{A_{1} \lambda \sqrt{-\Omega}\left(a_{1} \Omega \sinh (\xi \sqrt{-\Omega})-\sqrt{a_{1}^{2} \Omega^{2}-\rho^{2}} \cosh (\xi \sqrt{-\Omega})\right)}{-\lambda \sqrt{a_{1}^{2} \Omega^{2}-\rho^{2}} \sinh (\xi \sqrt{-\Omega})+a_{1} \lambda \Omega \cosh (\xi \sqrt{-\Omega})+\rho \Omega} e^{i P} . \\
q_{5}=A_{1}\left(\frac{\lambda \sqrt{-\Omega}\left(a_{1} \Omega \sinh (\xi \sqrt{-\Omega})-\sqrt{a_{1}^{2} \Omega^{2}-\rho^{2}} \cosh (\xi \sqrt{-\Omega})\right)}{-\lambda \sqrt{a_{1}^{2} \Omega^{2}-\rho^{2}} \sinh (\xi \sqrt{-\Omega})+a_{1} \lambda \Omega \cosh (\xi \sqrt{-\Omega})+\rho \Omega} \frac{\sqrt{2 \rho-\Omega^{2}}}{\sqrt{\Omega}}\right) e^{i P} . \\
q_{6}=B_{0}\left(\frac{\lambda \Omega}{a_{2} \lambda \sinh (\xi \sqrt{-\Omega})+a_{1} \lambda \cosh (\xi \sqrt{-\Omega})+\rho}+\frac{6}{2 \rho}\right) e^{i P} .
\end{gathered}
$$

Case 3: If $\Omega=0$,

\section{Set 1:}

$$
\begin{aligned}
& A_{0}=0, B_{0}= \pm \sqrt{A_{1}^{2}\left(a_{1}^{2}-2 a_{2}^{2} \rho\right)}, k_{3}=\frac{1}{2}\left(\frac{3 \alpha_{2} \sqrt{A_{1}^{2}(8 \lambda v+4)-(4 \eta v-1)\left(k_{1}^{2}+\omega^{2}\right)}}{\sqrt{4 \eta v-1}}-\alpha_{1} k_{1}-2 \tau \omega\right), \\
& \alpha_{3}=\frac{\alpha_{2}^{2}(4 \eta v-1)-\alpha_{1}^{2}+4 \gamma v+\tau^{2}(4 \eta v-1)}{2}, k_{2}=-\frac{\sqrt{A_{1}^{2}(8 \lambda v+4)-(4 \eta v-1)\left(k_{1}^{2}+\omega^{2}\right)}}{\sqrt{4 \eta v-1}} .
\end{aligned}
$$

\section{Set 2:}

$$
\begin{aligned}
& A_{0}=B_{0}=0, a_{1}=\sqrt{2} a_{2} \sqrt{\rho}, \alpha_{3}=\frac{1}{2}\left(\alpha_{2}^{2}(4 \eta v-1)-\alpha_{1}^{2}+4 \gamma v+\tau^{2}(4 \eta v-1)\right), \\
& k_{2}= \pm \frac{\sqrt{A_{1}^{2}(8 \lambda v+4)-(4 \eta v-1)\left(k_{1}^{2}+\omega^{2}\right)}}{\sqrt{4 \eta v-1}}, \\
& k_{3}=\frac{1}{2}\left(\mp \frac{3 \alpha_{2} \sqrt{A_{1}^{2}(8 \lambda v+4)-(4 \eta v-1)\left(k_{1}^{2}+\omega^{2}\right)}}{\sqrt{4 \eta v-1}}-\alpha_{1} k_{1}-2 \tau \omega\right) .
\end{aligned}
$$


Set 3:

$$
\begin{aligned}
& A_{0}=B_{0}=0, \alpha_{3}=\frac{1}{2}, \alpha_{2}^{2}(4 \eta v-1)-\alpha_{1}^{2}+4 \gamma v+\tau^{2}(4 \eta v-1), k_{2}=\mp \frac{\sqrt{A_{1}^{2}(8 \lambda v+4)-(4 \eta v-1)\left(k_{1}^{2}+\omega^{2}\right)}}{\sqrt{4 \eta v-1}}, \\
& k_{3}=\frac{1}{2}\left( \pm \frac{3 \alpha_{2} \sqrt{A_{1}^{2}(8 \lambda v+4)-(4 \eta v-1)\left(k_{1}^{2}+\omega^{2}\right)}}{\sqrt{4 \eta v-1}}-\alpha_{1} k_{1}-2 \tau \omega\right), a_{1}=-\sqrt{2} a_{2} \sqrt{\rho} .
\end{aligned}
$$

Solitons solutions of Equation (1) from solutions (35), (36) and (37) are constructed as

$$
\begin{gathered}
q_{7}(\xi)=\frac{2\left(A_{1}\left(a_{1}+\xi \rho\right) \pm \sqrt{A_{1}^{2}\left(a_{1}^{2}-2 a_{2}^{2} \rho\right)}\right)}{2 a_{1} \xi+2 a_{2}+\xi^{2} \rho} e^{i P} . \\
q_{8}(\xi)=\frac{2 A_{1}\left(\sqrt{2} a_{2} \sqrt{\rho}+\xi \rho\right)}{2 a_{2}(\sqrt{2} \xi \sqrt{\rho}+1)+\xi^{2} \rho} e^{i P} . \\
q_{9}=\frac{2 A_{1}\left(\xi \rho-\sqrt{2} a_{2} \sqrt{\rho}\right)}{a_{2}(2-2 \sqrt{2} \xi \sqrt{\rho})+\xi^{2} \rho} e^{i P} .
\end{gathered}
$$

\subsection{Solitons Solutions by the $\operatorname{Exp}(-\phi(\xi))$-Expansion Method}

In this sub-part, the $\exp (\phi(\xi))$-expansion method is employed to construct the solution of Equation (1). Apply the homogeneous balance principle on Equation (21) and assume the solution as

$$
\psi(\xi)=\frac{B_{-1}}{\exp (-\phi(\xi))}+B_{0}+B_{1} \exp (-\phi(\xi))
$$

Substituting Equation (41) along Equation (10) in Equation (21) and setting the coefficients to zero of $(\exp (-\phi(x)))^{i}$, we obtain a set of equations in parameters $B_{-1}, B_{0}, B_{1}, \lambda, \eta, a, b$ and $v$. This algebraic set of equations are solved by utilizing Mathematica 9 . The following sets are achieved as

Set 1:

$$
\begin{aligned}
B_{-1}= & 0, B_{0}=\frac{b B_{1}}{2}, k_{3}=\frac{-\alpha_{1} k_{1}-3 \alpha_{2} k_{2}-2 \tau \omega}{2}, \lambda=\frac{(4 \eta v-1)\left(k_{1}^{2}+k_{2}^{2}+\omega^{2}\right)-B_{1}^{2}}{2 B_{1}^{2} v}, \\
\alpha_{3}= & \frac{1}{4}\left(-k_{1}^{2}\left(4 a-b^{2}\right)(4 \eta v-1)-k_{2}^{2}\left(4 a-b^{2}\right)(4 \eta v-1)-16 a \eta v \omega^{2}+8 \alpha_{2}^{2} \eta v+4 a \omega^{2}\right. \\
& \left.-2 \alpha_{1}^{2}-2 \alpha_{2}^{2}+4 b^{2} \eta v \omega^{2}-b^{2} \omega^{2}+8 \gamma v+8 \eta v \tau^{2}-2 \tau^{2}\right) .
\end{aligned}
$$

Set 2:

$$
\begin{aligned}
B_{0}= & \frac{b B_{-1}}{2 a}, B_{1}=0, k_{3}=\frac{-\alpha_{1} k_{1}-3 \alpha_{2} k_{2}-2 \tau \omega}{2}, \lambda=\frac{a^{2}(4 \eta v-1)\left(k_{1}^{2}+k_{2}^{2}+\omega^{2}\right)-B_{-1}^{2}}{2 B_{-1}^{2} v}, \\
\alpha_{3}= & \frac{1}{4}\left(-k_{1}^{2}\left(4 a-b^{2}\right)(4 \eta v-1)-k_{2}^{2}\left(4 a-b^{2}\right)(4 \eta v-1)-16 a \eta v \omega^{2}+8 \alpha_{2}^{2} \eta v+4 a \omega^{2}-2 \alpha_{1}^{2}\right. \\
& \left.-2 \alpha_{2}^{2}+4 b^{2} \eta v \omega^{2}-b^{2} \omega^{2}+8 \gamma v+8 \eta v \tau^{2}-2 \tau^{2}\right) .
\end{aligned}
$$

Set 3:

$$
\begin{aligned}
& k_{3}=-\frac{\alpha_{1} k_{1}}{2}-\frac{3 \alpha_{2} k_{2}}{2}+\sqrt{-k_{1}^{2}-k_{2}^{2}} \tau, \alpha_{3}=\frac{\alpha_{2}^{2}(4 \eta v-1)-\alpha_{1}^{2}+4 \gamma v+4 \eta v \tau^{2}-\tau^{2}}{2}, \\
& \lambda=-\frac{1}{2 v}, \omega=-\sqrt{-k_{1}^{2}-k_{2}^{2}} .
\end{aligned}
$$


Set 4:

$$
\begin{aligned}
& B_{-1}=0, k_{3}=-\frac{\alpha_{1} k_{1}}{2}-\frac{3 \alpha_{2} k_{2}}{2}+\sqrt{-k_{1}^{2}-k_{2}^{2}} \tau, \alpha_{3}=\frac{\alpha_{2}^{2}(4 \eta v-1)-\alpha_{1}^{2}+4 \gamma v+4 \eta v \tau^{2}-\tau^{2}}{2}, \\
& \lambda=-\frac{1}{2 v}, \omega= \pm \sqrt{-k_{1}^{2}-k_{2}^{2}} .
\end{aligned}
$$

Set 5:

$$
\begin{aligned}
& B_{1}=0, k_{3}=-\frac{\alpha_{1} k_{1}}{2}-\frac{3 \alpha_{2} k_{2}}{2}+\sqrt{-k_{1}^{2}-k_{2}^{2}} \tau, \alpha_{3}=\frac{\alpha_{2}^{2}(4 \eta v-1)-\alpha_{1}^{2}+4 \gamma v+4 \eta v \tau^{2}-\tau^{2}}{2}, \\
& \lambda=-\frac{1}{2 v}, \omega=-\sqrt{-k_{1}^{2}-k_{2}^{2}} .
\end{aligned}
$$

Set 6:

$$
\begin{aligned}
B_{-1}= & \frac{2 a B_{0}}{b}, B_{1}=0, \alpha_{1}=-\frac{3 \alpha_{2} k_{2}+2 k_{3}+2 \tau \omega}{k_{1}}, \lambda=\frac{b^{2}(4 \eta v-1)\left(k_{1}^{2}+k_{2}^{2}+\omega^{2}\right)-4 B_{0}^{2}}{8 B_{0}^{2} v}, \\
\alpha_{3}= & \left(k _ { 1 } ^ { 2 } \left(-k_{2}^{2}\left(4 a-b^{2}\right)(4 \eta v-1)-16 a \eta v \omega^{2}+\alpha_{2}^{2}(8 \eta v-2)+4 a \omega^{2}+4 b^{2} \eta v \omega^{2}-b^{2} \omega^{2}+8 \gamma v\right.\right. \\
& \left.\left.+8 \eta v \tau^{2}-2 \tau^{2}\right)+k_{1}^{4}\left(-\left(4 a-b^{2}\right)\right)(4 \eta v-1)-2\left(3 \alpha_{2} k_{2}+2 k_{3}+2 \tau \omega\right)^{2}\right) / 4 k_{1}^{2} .
\end{aligned}
$$

Set 7:

$$
\begin{aligned}
B_{-1} & =0, B_{0}=\frac{b B_{1}}{2}, \alpha_{1}=-\frac{3 \alpha_{2} k_{2}+2 k_{3}+2 \tau \omega}{k_{1}}, \lambda=\frac{(4 \eta v-1)\left(k_{1}^{2}+k_{2}^{2}+\omega^{2}\right)-B_{1}^{2}}{2 B_{1}^{2} v}, \\
\alpha_{3}= & \left(k _ { 1 } ^ { 2 } \left(-k_{2}^{2}\left(4 a-b^{2}\right)(4 \eta v-1)-16 a \eta v \omega^{2}+\alpha_{2}^{2}(8 \eta v-2)+4 a \omega^{2}+4 b^{2} \eta v \omega^{2}-b^{2} \omega^{2}\right.\right. \\
& \left.\left.+8 \gamma v+8 \eta v \tau^{2}-2 \tau^{2}\right)+k_{1}^{4}\left(-\left(4 a-b^{2}\right)\right)(4 \eta v-1)-2\left(3 \alpha_{2} k_{2}+2 k_{3}+2 \tau \omega\right)^{2}\right) / 4 k_{1}^{2} .
\end{aligned}
$$

From set 1, the following five types of solutions of Equation (1) are constructed as

Type I: When $a \neq 0, b^{2}-4 a>0$,

$$
q_{1}(\xi)=\frac{B_{1}}{2}\left(b-\frac{4 a}{\sqrt{b^{2}-4 a} \tanh \left(\frac{\sqrt{b^{2}-4 a}}{2}(\xi+c)\right)+b}\right) e^{i P} .
$$

Type II When $a \neq 0, b^{2}-4 a<0$,

$$
q_{2}(\xi)=\frac{B_{1}}{2}\left(b-\frac{4 a}{b-\sqrt{4 a-b^{2}} \tan \left(\frac{\sqrt{4 a-b^{2}}}{2}(\xi+c)\right)}\right) e^{i P} .
$$

Type III When $a=0, b \neq 0 \& b^{2}-4 a>0$,

$$
q_{3}(\xi)=\frac{b B_{1}}{2}\left(\frac{2}{e^{b(\xi+c)}-1}+1\right) e^{i P} .
$$

Type IV When $a \neq, b \neq 0 \& b^{2}-4 a=0$,

$$
q_{4}(\xi)=\frac{b B_{1}}{2 b(\xi+c)+2} e^{i P} .
$$


Type V When $a=0,=0 \& b^{2}-4 a=0$,

$$
q_{5}(\xi)=\frac{B_{1}}{2}\left(b+\frac{2}{\xi+c}\right) e^{i P} .
$$

Similarly, further novel exact solutions of (1) forming other solution sets can be constructed.

\subsection{Solitons Solutions by the Proposed F-Expansion Method}

In this sub-part, the proposed F-Expansion technique is employed to construct the solitons and wave solutions of Equation (1). Apply the homogeneous balance principle on Equation (21) and assume the solution of Equation (21) as

$$
\psi(\xi)=A_{0}+A_{1}(a+F(\xi))+\frac{B_{1}}{a+F(\xi)} .
$$

Substituting Equation (54) along Equation (17) in Equation (21) and setting the coefficients to zero of $\frac{F^{j}(\xi)}{(a+F(\xi))^{k}}$, we obtain a set of equations in parameters $A_{0}, A_{1}, B_{1}, d_{0}, d_{1}, d_{2}, d_{3}$, and $v$. This algebraic set of equations are solved by utilizing Mathematica 9. The following cases of solutions are achieved as

Case 1: If $d_{0}=d_{2}=0$,

$$
\begin{aligned}
& A_{0}=-a A_{1}, B_{1}=0, \alpha_{3}=\frac{d_{3}\left(\gamma-\alpha_{1}^{2} \eta\right)-A_{1}^{2} d_{1}(2 \eta+\lambda)}{2 d_{3} \eta}, v=\frac{1}{4 \eta^{\prime}}, \\
& k_{3}=-\frac{A_{1}^{2}(2 \eta+\lambda)+4 d_{3} \eta^{2}\left(\alpha_{1} k_{1}+3 \alpha_{2} k_{2}+2 \tau \omega\right)}{8 d_{3} \eta^{2}} . \\
& A_{0}=B_{1}=a=0, k_{3}=-\frac{A_{1}^{2}(2 \eta+\lambda)+4 d_{3} \eta^{2}\left(\alpha_{1} k_{1}+3 \alpha_{2} k_{2}+2 \tau \omega\right)}{8 d_{3} \eta^{2}}, \\
& v=\frac{1}{4 \eta}, \alpha_{3}=\frac{d_{3}\left(\gamma-\alpha_{1}^{2} \eta\right)-A_{1}^{2} d_{1}(2 \eta+\lambda)}{2 d_{3} \eta} .
\end{aligned}
$$

The solitons solutions of Equation (1) from solutions (55) and (56) are constructed as

$$
\begin{array}{ll}
q_{11}(\xi)=\frac{A_{1} \sqrt{d_{1}} e^{d_{1} \xi}}{\sqrt{1-d_{3} e^{2 d_{1} \xi}}} e^{i\left(\alpha_{1} x+\alpha_{2} y+\alpha_{3} z+t \tau+\epsilon\right)}, & d_{1}>0 . \\
q_{12}(\xi)=\frac{A_{1} \sqrt{-d_{1}}}{\sqrt{e^{-2 d_{1} \xi}+d_{3}}} e^{i\left(\alpha_{1} x+\alpha_{2} y+\alpha_{3} z+t \tau+\epsilon\right)}, & d_{1}<0 . \\
q_{13}(\xi)=\frac{A_{1} \sqrt{d_{1}} e^{d_{1} \xi}}{\sqrt{1-d_{3} e^{2 d_{1} \xi}}} e^{i\left(\alpha_{1} x+\alpha_{2} y+\alpha_{3} z+t \tau+\epsilon\right)}, & d_{1}>0 . \\
q_{14}(\xi)=\frac{A_{1} \sqrt{-d_{1}}}{\sqrt{e^{-2 d_{1} \xi}+d_{3}}} e^{i\left(\alpha_{1} x+\alpha_{2} y+\alpha_{3} z+t \tau+\epsilon\right)}, & d_{1}<0 .
\end{array}
$$


Case 2: If $d_{0}=d_{3}=0$,

$$
\begin{gathered}
A_{0}=0, B_{1}=0, \alpha_{3}=\frac{A_{1}^{2} d_{1}^{2}(2 \lambda v+1)+2 d_{2}^{2}\left(\alpha_{2}^{2}(4 \eta v-1)-\alpha_{1}^{2}+4 \gamma v+\tau^{2}(4 \eta v-1)\right)}{4 d_{2}^{2}},{ }^{2} \frac{d_{1}}{2 d_{2}}, k_{3}=\frac{1}{2}\left(\frac{3 \alpha_{2} \sqrt{A_{1}^{2}(2 \lambda v+1)-d_{2}^{2}(4 \eta v-1)\left(k_{1}^{2}+\omega^{2}\right)}}{\sqrt{d_{2}^{2}(4 \eta v-1)}}-\alpha_{1} k_{1}-2 \tau \omega\right), \\
k_{2}=-\frac{\sqrt{A_{1}^{2}(2 \lambda v+1)-d_{2}^{2}(4 \eta v-1)\left(k_{1}^{2}+\omega^{2}\right)}}{\sqrt{d_{2}^{2}(4 \eta v-1)}} . \\
A_{0}=0, B_{1}=-\frac{A_{1} d_{1}^{2}}{4 d_{2}^{2}}, \alpha_{3}=\frac{d_{2}^{2}\left(\alpha_{2}^{2}(4 \eta v-1)-\alpha_{1}^{2}+4 \gamma v+\tau^{2}(4 \eta v-1)\right)-A_{1}^{2} d_{1}^{2}(2 \lambda v+1)}{2 d_{2}^{2}} \\
a=\frac{d_{1}}{2 d_{2}}, k_{3}=\frac{1}{2}\left(\frac{3 \alpha_{2} \sqrt{A_{1}^{2}(2 \lambda v+1)-d_{2}^{2}(4 \eta v-1)\left(k_{1}^{2}+\omega^{2}\right)}}{\sqrt{d_{2}^{2}(4 \eta v-1)}}-\alpha_{1} k_{1}-2 \tau \omega\right) \\
k_{2}=-\frac{\sqrt{A_{1}^{2}(2 \lambda v+1)-d_{2}^{2}(4 \eta v-1)\left(k_{1}^{2}+\omega^{2}\right)}}{\sqrt{d_{2}^{2}(4 \eta v-1)}} . \\
A_{0}=\frac{A_{1}}{2}\left(\frac{d_{1}}{d_{2}}-2 a\right), \alpha_{3}=\frac{A_{1}^{2} d_{1}^{2}(2 \lambda v+1)+2 d_{2}^{2}\left(\alpha_{2}^{2}(4 \eta v-1)-\alpha_{1}^{2}+4 \gamma v+\tau^{2}(4 \eta v-1)\right)}{4 d_{2}^{2}} \\
B_{1}=0, k_{3}=\frac{1}{2}\left(\frac{3 \alpha_{2} \sqrt{A_{1}^{2}(2 \lambda v+1)-d_{2}^{2}(4 \eta v-1)\left(k_{1}^{2}+\omega^{2}\right)}}{\sqrt{d_{2}^{2}(4 \eta v-1)}}-\alpha_{1} k_{1}-2 \tau \omega\right) \\
\sqrt{A_{2}^{2}(2 \lambda \nu v-1)}
\end{gathered}
$$

The solitons of Equation (1) from solutions (61) and (62) are constructed as

$$
\begin{gathered}
q_{21}(\xi)=\frac{A_{1} d_{1}\left(d_{2} e^{d_{1} \xi}+1\right)}{2 d_{2}\left(1-d_{2} e^{d_{1} \xi}\right)} e^{i\left(\alpha_{1} x+\alpha_{2} y+\alpha_{3} z+t \tau+\epsilon\right)}, \quad d_{1}>0 . \\
q_{22}(\xi)=\frac{A_{1} d_{1}\left(1-d_{2} e^{d_{1} \xi}\right)}{2 d_{2}\left(d_{2} e^{d_{1} \xi}+1\right)} e^{i\left(\alpha_{1} x+\alpha_{2} y+\alpha_{3} z+t \tau+\epsilon\right)}, \quad d_{1}<0 . \\
q_{23}(\xi)=\frac{2 A_{1} d_{1} e^{d_{1} \xi}}{1-d_{2}^{2} e^{2 d_{1} \xi}} e^{i\left(\alpha_{1} x+\alpha_{2} y+\alpha_{3} z+t \tau+\epsilon\right)}, \quad d_{1}>0 . \\
q_{24}(\xi)=\frac{2 A_{1} d_{1} e^{d_{1} \xi}}{d_{2}^{2} e^{2 d_{1} \xi}-1} e^{i\left(\alpha_{1} x+\alpha_{2} y+\alpha_{3} z+t \tau+\epsilon\right)}, \quad d_{1}<0 .
\end{gathered}
$$

Similarly, more generalized results can be constructed of Equation (1) from solution (63). 
Case 3: If $d_{1}=d_{3}=0$,

$$
\begin{gathered}
A_{0}=-a A_{1}, \alpha_{3}=\frac{d_{2}\left(\alpha_{2}^{2}(4 \eta v-1)-\alpha_{1}^{2}+4 \gamma v+\tau^{2}(4 \eta v-1)\right)-2 A_{1}^{2} d_{0}(2 \lambda v+1)}{2 d_{2}}, k_{1}=-\frac{1}{2}\left(\frac{3 \alpha_{2} \sqrt{A_{1}^{2}(2 \lambda v+1)-d_{2}^{2}(4 \eta v-1)\left(k_{1}^{2}+\omega^{2}\right)}}{\sqrt{d_{2}^{2}(4 \eta v-1)}}+\alpha_{1} k_{1}+2 \tau \omega\right), \\
B_{1}=\frac{\sqrt{A_{1}^{2}(2 \lambda v+1)-d_{2}^{2}(4 \eta v-1)\left(k_{1}^{2}+\omega^{2}\right)}}{\sqrt{d_{2}^{2}(4 \eta v-1)}} . \\
A_{0}=0, a=0, \alpha_{3}=\frac{d_{2}\left(\alpha_{2}^{2}(4 \eta v-1)-\alpha_{1}^{2}+4 \gamma v+\tau^{2}(4 \eta v-1)\right)-8 A_{1}^{2} d_{0}(2 \lambda v+1)}{2 d_{2}}, \\
B_{1}=-\frac{A_{1} d_{0}}{d_{2}}, k_{3}=\frac{1}{2}\left(\frac{3 \alpha_{2} \sqrt{A_{1}^{2}(2 \lambda v+1)-d_{2}^{2}(4 \eta v-1)\left(k_{1}^{2}+\omega^{2}\right)}}{\sqrt{d_{2}^{2}(4 \eta v-1)}}-\alpha_{1} k_{1}-2 \tau \omega\right), \\
k_{2}=-\frac{\sqrt{A_{1}^{2}(2 \lambda v+1)-d_{2}^{2}(4 \eta v-1)\left(k_{1}^{2}+\omega^{2}\right)}}{\sqrt{d_{2}^{2}(4 \eta v-1)}}, \\
k_{3}=\frac{1}{2}\left(\frac{3 \alpha_{2} \sqrt{A_{1}^{2}(2 \lambda v+1)-d_{2}^{2}(4 \eta v-1)\left(k_{1}^{2}+\omega^{2}\right)}}{\left.\sqrt{d_{2}^{2}(4 \eta v-1)}-\alpha_{1} k_{1}-2 \tau \omega\right) .}\right. \\
A_{0}=0, \alpha_{3}=\frac{d_{2}\left(\alpha_{2}^{2}(4 \eta v-1)-\alpha_{1}^{2}+4 \gamma v+\tau^{2}(4 \eta v-1)\right)-2 A_{1}^{2} d_{0}(2 \lambda v+1)}{2 d_{2}}, \\
B_{1}=0, a=0, k_{2}=-\frac{\sqrt{A_{1}^{2}(2 \lambda v+1)-d_{2}^{2}(4 \eta v-1)\left(k_{1}^{2}+\omega^{2}\right)}}{2 \eta \eta-1)}
\end{gathered}
$$

The solitons solutions of Equation (1) from solutions (68) and (69) are constructed as

$$
\begin{gathered}
q_{31}(\xi)=\frac{A_{1} d_{0} \tan \left(\sqrt{d_{0} d_{2}} \xi\right)}{\sqrt{d_{0} d_{2}}} e^{i\left(\alpha_{1} x+\alpha_{2} y+\alpha_{3} z+t \tau+\epsilon\right)}, \quad d_{0} d_{2}>0 . \\
q_{32}(\xi)=\frac{A_{1} d_{0} \tanh \left(\sqrt{-d_{0} d_{2}} \xi\right)}{\sqrt{-d_{0} d_{2}}} e^{i\left(\alpha_{1} x+\alpha_{2} y+\alpha_{3} z+t \tau+\epsilon\right)}, \quad d_{0} d_{2}<0 . \\
q_{33}(\xi)=\frac{A_{1} d_{0} \tan \left(\sqrt{d_{0} d_{2}} \xi\right)\left(1-\cot ^{2}\left(\sqrt{d_{0} d_{2}} \xi\right)\right)}{\sqrt{d_{0} d_{2}}} e^{i\left(\alpha_{1} x+\alpha_{2} y+\alpha_{3} z+t \tau+\epsilon\right)}, \quad d_{0} d_{2}>0 . \\
q_{34}(\xi)=\frac{A_{1} d_{0} \tanh \left(\sqrt{-d_{0} d_{2}} \xi\right)\left(\operatorname{coth}^{2}\left(\sqrt{-d_{0} d_{2}} \xi\right)+1\right)}{\sqrt{-d_{0} d_{2}}} e^{i\left(\alpha_{1} x+\alpha_{2} y+\alpha_{3} z+t \tau+\epsilon\right)}, \quad d_{0} d_{2}<0 .
\end{gathered}
$$

Similarly, more results can be constructed of Equation (1) from solutions (63). 
Case 4: If $d_{3}=0$,

$$
\begin{aligned}
A_{0}= & \frac{B_{1}\left(d_{1}-2 a d_{2}\right)}{2\left(a\left(a d_{2}-d_{1}\right)+d_{0}\right)}, k_{2}=-\frac{\sqrt{B_{1}^{2}(2 \lambda v+1)-\left(a\left(a d_{2}-d_{1}\right)+d_{0}\right)^{2}(4 \eta v-1)\left(k_{1}^{2}+\omega^{2}\right)}}{\sqrt{\left(a\left(a d_{2}-d_{1}\right)+d_{0}\right)^{2}(4 \eta v-1)}}, \\
A_{1}= & 0, k_{3}=\frac{1}{2}\left(\frac{3 \alpha_{2} \sqrt{B_{1}^{2}(2 \lambda v+1)-\left(a^{2} d_{2}-a d_{1}+d_{0}\right)^{2}(4 \eta v-1)\left(k_{1}^{2}+\omega^{2}\right)}}{\sqrt{\left(a\left(a d_{2}-d_{1}\right)+d_{0}\right)^{2}(4 \eta v-1)}}-\alpha_{1} k_{1}-2 \tau \omega\right), \\
\alpha_{3}= & \left(2 a^{4} d_{2}^{2}\left(\alpha_{2}^{2}(4 \eta v-1)-\alpha_{1}^{2}+4 \gamma v+\tau^{2}(4 \eta v-1)\right)+4 a^{3} d_{1} d_{2}\left(\alpha_{2}^{2}(1-4 \eta v)+\alpha_{1}^{2}-4 v\left(\gamma+\eta \tau^{2}\right)\right.\right. \\
& \left.+\tau^{2}\right)+d_{1}^{2}\left(2 a^{2}\left(\alpha_{2}^{2}(4 \eta v-1)-\alpha_{1}^{2}+4 \gamma v+\tau^{2}(4 \eta v-1)\right)+B_{1}^{2}(2 \lambda v+1)\right)+4 d_{0}\left(d _ { 2 } \left(a ^ { 2 } \left(\alpha_{2}^{2}(4 \eta v\right.\right.\right. \\
& \left.\left.-1)-\alpha_{1}^{2}+4 \gamma v+\tau^{2}(4 \eta v-1)\right)-B_{1}^{2}(2 \lambda v+1)\right)+a d_{1}\left(\alpha_{2}^{2}(1-4 \eta v)+\alpha_{1}^{2}-4 v\left(\gamma+\eta \tau^{2}\right)\right. \\
& \left.\left.\left.+\tau^{2}\right)\right)+d_{0}^{2}\left(\alpha_{2}^{2}(8 \eta v-2)-2 \alpha_{1}^{2}+8 \gamma v+2 \tau^{2}(4 \eta v-1)\right)\right) /\left(4\left(a\left(a d_{2}-d_{1}\right)+d_{0}\right)^{2}\right) . \\
A_{0}= & \frac{A_{1}\left(d_{1}-2 a d_{2}\right)-\sqrt{A_{1}^{2}\left(d_{1}^{2}-4 d_{0} d_{2}\right)}}{2 d_{2}}, k_{2}=-\frac{\sqrt{A_{1}^{2}(2 \lambda v+1)-d_{2}^{2}(4 \eta v-1)\left(k_{1}^{2}+\omega^{2}\right)}}{\sqrt{d_{2}^{2}(4 \eta v-1)}}, \\
B_{1}= & 0, \alpha_{3}=\frac{2 A_{1}^{2}\left(d_{1}^{2}-4 d_{0} d_{2}\right)(2 \lambda v+1)+d_{2}^{2}\left(\alpha_{2}^{2}(4 \eta v-1)-\alpha_{1}^{2}+4 \gamma v+\tau^{2}(4 \eta v-1)\right)}{2 d_{2}^{2}}, \\
k_{3}= & \left(3 \left(4 \alpha_{2} \eta d_{2} \sqrt{(4 \eta v-1)\left(A_{1}^{2}(2 \lambda v+1)-d_{2}^{2}(4 \eta v-1)\left(k_{1}^{2}+\omega^{2}\right)\right)}+A_{1}^{2} \sqrt{d_{1}^{2}-4 d_{0} d_{2}}(4 \eta v\right.\right. \\
& \left.-1)(2 \lambda v+1))-4 d_{2}^{2} \eta(4 \eta v-1)\left(\alpha_{1} k_{1}+2 \tau \omega\right)\right) /\left(8 d_{2}^{2} \eta(4 \eta v-1)\right) .
\end{aligned}
$$

The solitons solutions of Equation (1) from solutions (75) and (76) are constructed as

$$
\begin{aligned}
& q_{41}(\xi)=\frac{B_{1} e^{i\left(\alpha_{1} x+\alpha_{2} y+\alpha_{3} z+t \tau+\epsilon\right)}}{2}\left(\frac{4 d_{2}}{2 a d_{2}+\sqrt{4 d_{0} d_{2}-d_{1}^{2}} \tan \left(\frac{\sqrt{4 d_{0} d_{2}-d_{1}^{2}}}{2} \xi\right)-d_{1}}\right. \\
& \left.+\frac{d_{1}-2 a d_{2}}{a^{2} d_{2}-a d_{1}+d_{0}}\right), 4 d_{0} d_{2}>d_{1}^{2} \text {. }
\end{aligned}
$$

$$
q_{42}(\xi)=\frac{A_{1} \sqrt{4 d_{0} d_{2}-d_{1}^{2}} \tan \left(\frac{\sqrt{4 d_{0} d_{2}-d_{1}^{2}}}{2} \xi\right)-A_{1} \sqrt{d_{1}^{2}-4 d_{0} d_{2}}}{2 d_{2}} e^{i\left(\alpha_{1} x+\alpha_{2} y+\alpha_{3} z+t \tau+\epsilon\right)}, 4 d_{0} d_{2}>d_{1}^{2} .
$$

\section{Modulation Instability}

Several nonlinear systems reveal instability that results in the modulation of the steady state owing to the connection among nonlinear and dispersive effects. We examine the MI of model (1) employing the standard linear stability analysis [3-5,41,43]. The solutions of Equation (1) in the steady-state form are as

$$
q(x, y, z, t)=\left(\sqrt{P_{o}}+A(x, y, z, t)\right) e^{i \phi(z)}, \phi(z)=\delta \epsilon P_{o} z
$$

where the $P_{o}$ optical power is normalized. Through using the analysis of linear stability, the perturbation $A(x, y, z, t)$ is examined. Using Equation (79) in Equation (1) and linearizing, we get

$$
\begin{array}{r}
2 i \frac{\partial A}{\partial z}+(1-2 i \eta)\left(\frac{\partial^{2} A}{\partial x^{2}}+\frac{\partial^{2} A}{\partial y^{2}}+\frac{\partial^{2} A}{\partial t^{2}}\right)+2\left(i \gamma+2 P_{o}-\delta \epsilon P_{o}-2 i \lambda P_{o}+3 i \mu P_{o}^{2}-3 v P_{o}^{2}\right) A \\
+2 P_{o}\left(1-i \lambda+2 i \mu P_{o}-2 v P_{o}\right) A^{*}=0
\end{array}
$$

The above Equation (80) can be resolved easily in wave number domain. However, as $A^{*}$ terms (which shows a complex conjugate), the Fourier terms at $\omega$ and $-\omega$ are coupled so we seek the solution of Equation (80) that has a form as follows:

$$
A(x, y, z, t)=\alpha_{1} e^{i\left(k_{1} x+k_{2} y+k_{3} z-\omega t\right)}+\alpha_{2} e^{-i\left(k_{1} x+k_{2} y+k_{3} z-\omega t\right)},
$$


where $k_{1}$ is normalized wave number and and $\omega$ is a frequency of $A(x, y, z, t)$. Substituting Equation (81) in Equation (80), we obtained a dispersion relation as follows:

$$
k_{3}= \pm \frac{\sqrt{-B\left((2 \eta+i)\left(k_{1}^{2}+k_{2}^{2}+\omega^{2}\right)+8 i \gamma \delta \lambda \epsilon P_{o}^{3}+2(\lambda+i) P_{o}-18 \mu \nu P_{o}^{4}-4(\mu+i v) P_{o}^{2}\right)}}{2} .
$$

where $B=(2 \eta+i)\left(k_{1}^{2}+k_{2}^{2}+\omega^{2}\right)+8 i \gamma \delta \lambda \epsilon P_{o}^{3}-2(\lambda+i) P_{o}-18 \mu v P_{o}^{4}+4(\mu+i v) P_{o}^{2}$. The above dispersion relation (82) illustrates that stability of steady state be contingent on self-phase and Raman scattering. $\quad-B\left((2 \eta+i)\left(k_{1}^{2}+k_{2}^{2}+\omega^{2}\right)+8 i \gamma \delta \lambda \epsilon P_{o}^{3}+2(\lambda+i) P_{o}-18 \mu v P_{o}^{4}-4(\mu+i v) P_{o}^{2}\right)>0$, which shows that the steady state is stable against slight perturbations. In other cases, it becomes unstable. If $-B\left((2 \eta+i)\left(k_{1}^{2}+k_{2}^{2}+\omega^{2}\right)+8 i \gamma \delta \lambda \epsilon P_{o}^{3}+2(\lambda+i) P_{o}-18 \mu v P_{o}^{4}-4(\mu+i v) P_{o}^{2}\right)<0$, it shows that $k_{3}$ is imaginary; meanwhile, the perturbation cultivates exponentially. One can see this straightforwardly for instances of MI when $-B\left((2 \eta+i)\left(k_{1}^{2}+k_{2}^{2}+\omega^{2}\right)+8 i \gamma \delta \lambda \epsilon P_{o}^{3}+2(\lambda+i) P_{o}-18 \mu v P_{o}^{4}-4(\mu+i v) P_{o}^{2}\right)<0$. Underneath this condition, the evolution rate of the MI gain spectrum $h(k)$ can be expressed as

$$
\begin{aligned}
h\left(k_{1}, k_{2}, \omega\right) & =2 \operatorname{Img}\left(k_{1}, k_{2}, \omega\right) \\
= & \sqrt{-B\left((2 \eta+i)\left(k_{1}^{2}+k_{2}^{2}+\omega^{2}\right)+8 i \gamma \delta \lambda \epsilon P_{o}^{3}+2(\lambda+i) P_{o}-18 \mu v P_{o}^{4}-4(\mu+i v) P_{o}^{2}\right)} .
\end{aligned}
$$

\section{Discussion of Results and Their Physical Interpretation}

Many achieved results of Equation (1) via a proposed modified extended simple equation, $\exp (-\phi(\xi))$-expansion, and proposed F-expansion methods that are novel and dissimilar from the constructed results of other techniques [21,30,42]. The main key point to obtain novel results of our proposed methods is the main body of new proposed solutions (5), (9), (16) and ODEs (6), (10), (17). Equations (6), (10) and (17) provide a distinct type of results-for example, trigonometric, hyperbolic trigonometric, rational functions and other solutions through giving dissimilar values of parameters in these ODEs. The exact wave results in the forms of dark-bright solitons, breather-type and multi solitons, kink and anti-kink waves, and periodic and trigonometric function solutions are achieved. The constructed solutions are exact and more general. The authors in [21] discussed the global existence and small dispersion limit and the authors in [30] constructed the approximate solution of this model. The researchers in [42] simulated the vortex tori solitons of this dynamical model. Consequently, our achieved results are innovative and have not been articulated previously.

We demonstrated three-dimensional and two-dimensional structures of some obtained results for the model (1). In order to observe the physical appearance of this model, the physical structures are described by giving appropriate values to the parameters. In Figure 1, the structures of results (26)-(28) are depicted at dissimilar values of parameters: Figure $1 \mathrm{~A}$ is a multi bright-dark soliton and its 2-dim is in Figure 1B, Figure 1C is a multi solitons interaction and its 2-dim is in Figure 1D, Figure 1E is a periodic soliton and its 2-dim is in Figure 1F. In Figure 2, the structures of results (32)-(34) are depicted at dissimilar values of parameters: Figure 2A is a dark soliton and its 2-dim is in Figure 2B, Figure $2 \mathrm{C}$ is a anti-kink soliton and its 2-dim is in Figure 2D, and Figure 1E is a Bright soliton and and its 2-dim is in Figure 1F. In Figure 3, the structures of results (49) and (50) are depicted at dissimilar values of parameters: Figure $3 \mathrm{~A}$ is a periodic solitary wave and its 2-dim is in Figure 3B, Figure 3C is a breather-type waves of strange structure and its 2-dim is in Figure 3D. 


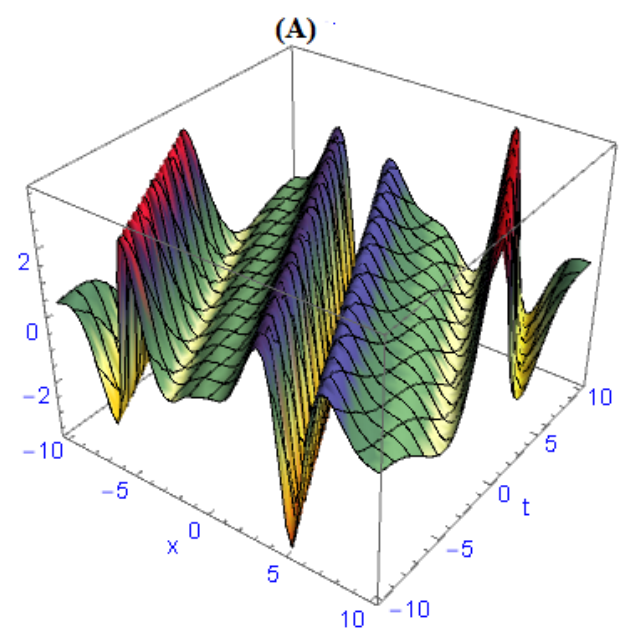

(B)
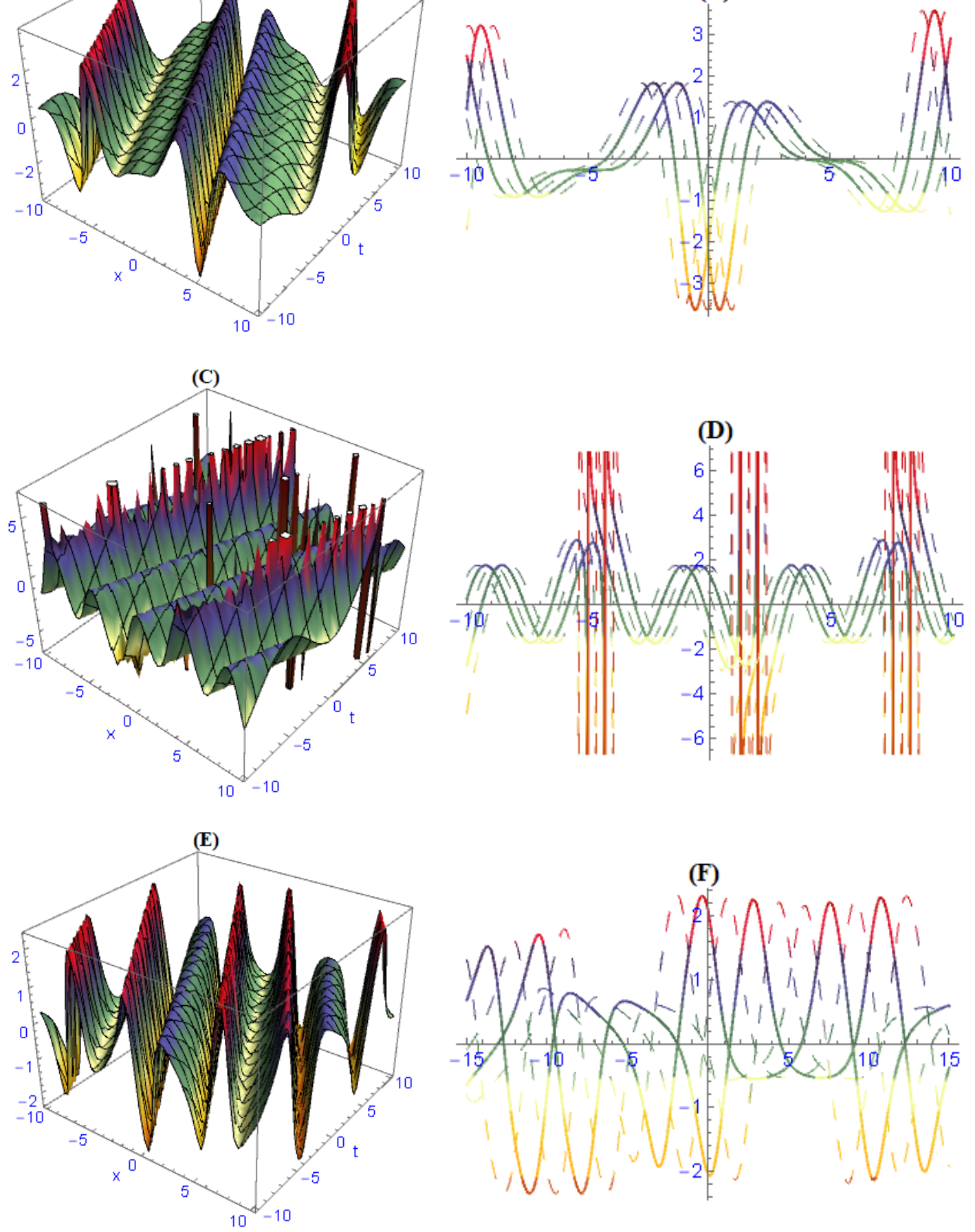

(F)

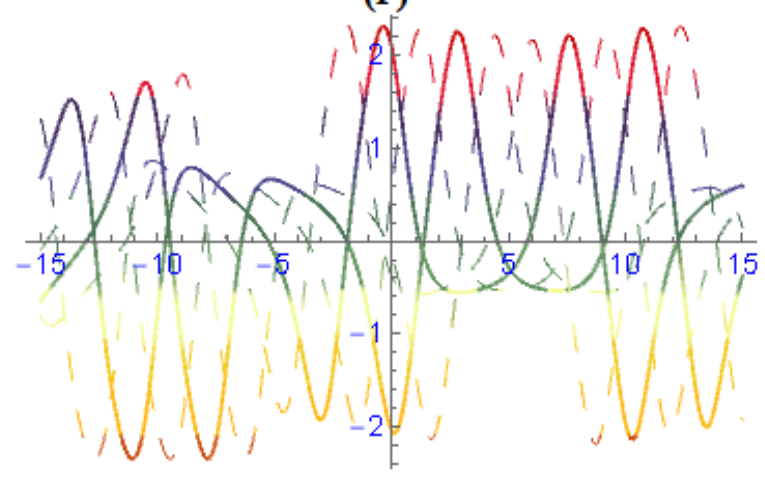

Figure 1. The graph of results (26)-(28) are depicted at dissimilar values of parameters and we obtained: (A) multi bright-dark solitons and its 2-dim in (B), (C) multi solitons interaction and its 2-dim in (D), (E) periodic solitons and and its 2-dim in (F). 

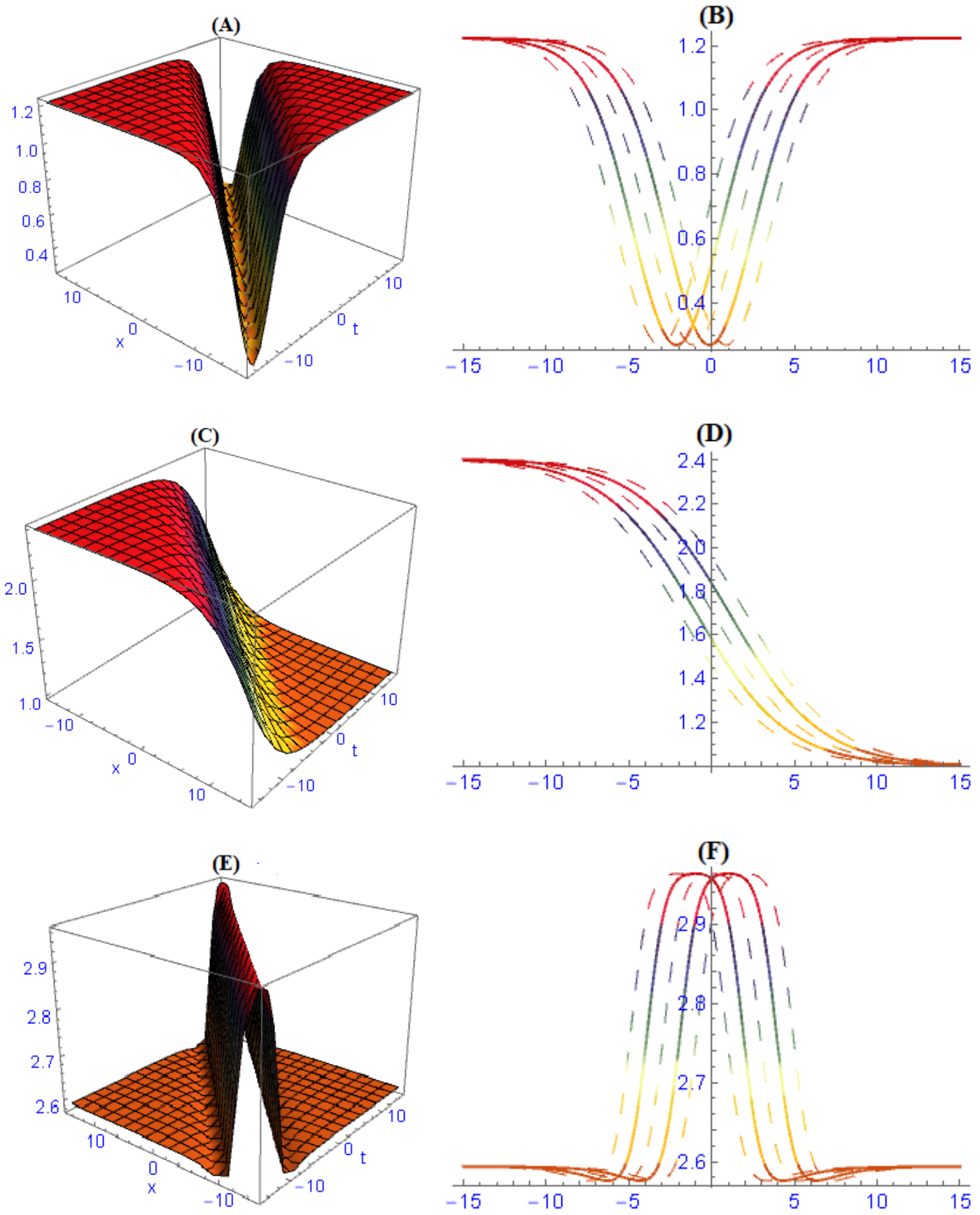

Figure 2. The graph of results (32)-(34) are depicted at dissimilar values of parameters and we obtained: (A) dark soliton and its 2-dim in (B), (C) anti-kink soliton and its 2-dim in (D), (E) bright soliton and and its 2-dim in (F). 

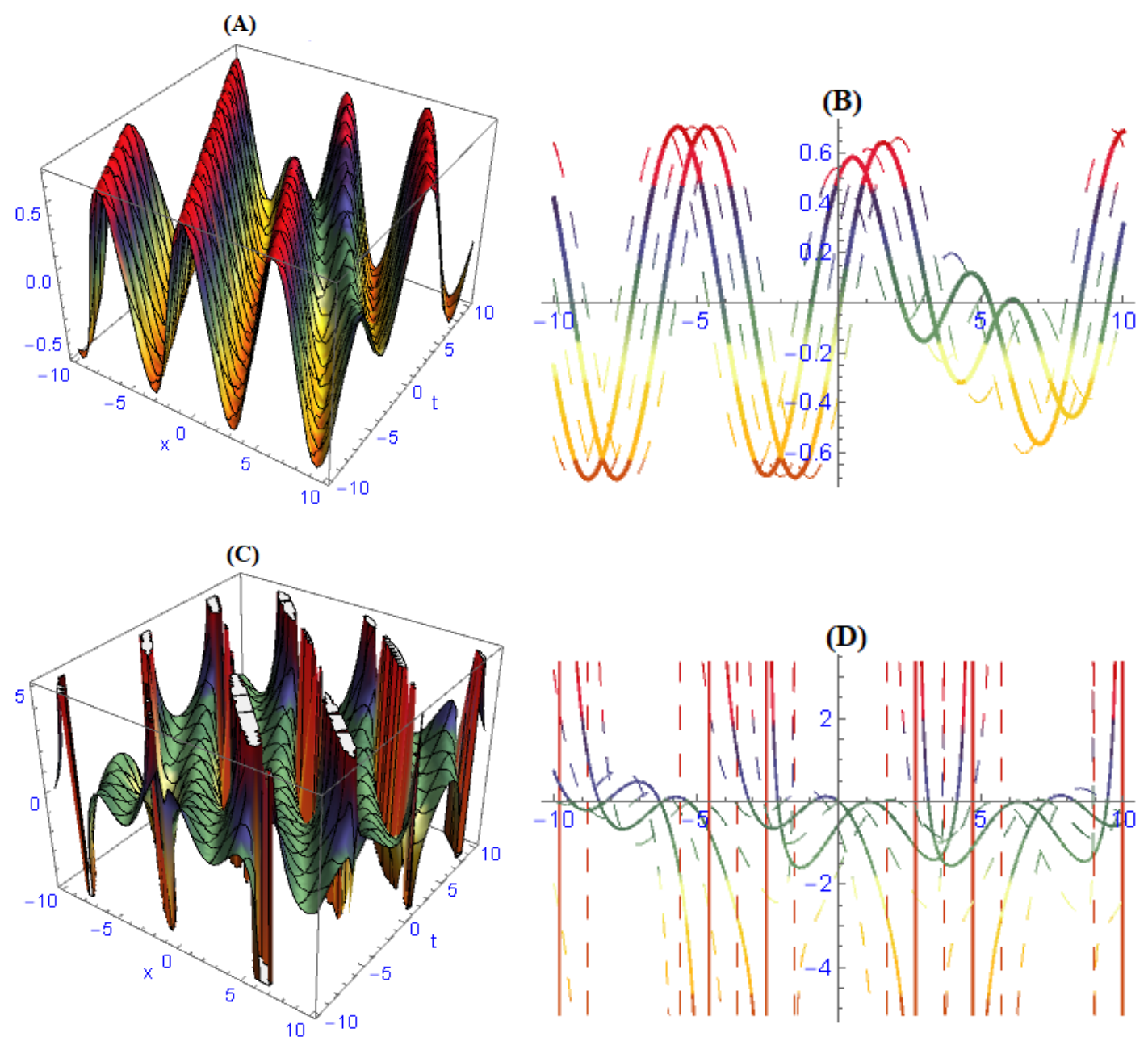

Figure 3. Solitary wave and soliton in various silhouettes are depicted of solutions (49) and (50) by choosing different values of parameters: (A) periodic solitary wave and its 2-dim in (B), (C) breather-type waves of strange structure and its 2-dim in (D).

In Figure 4, the structures of results (57) and (59) are depicted at dissimilar values of parameters: Figure $4 \mathrm{~A}$ is a bright solitary wave and its 2-dim is in Figure 4B, Figure 4C is an anti-kink soliton and its 2-dim is in Figure 4D. The structures in Figure 5 are depicted of results (64) and (67) at dissimilar values of parameters: Figure 5A is a dark compact type soliton and its 2-dim is in Figure 5B, Figure 5C is a compact type soliton and its 2-dim is in Figure 5D. In Figure 6, the structures are depicted of results (73) and (78) at dissimilar values of parameters: Figure 6A is a periodic soliton and its 2-dim is in Figure 6B, Figure 6C is a multi-kink type solitary wave and its 2-dim is in Figure 6D. Figure 7 is the shape of dispersion relation. 

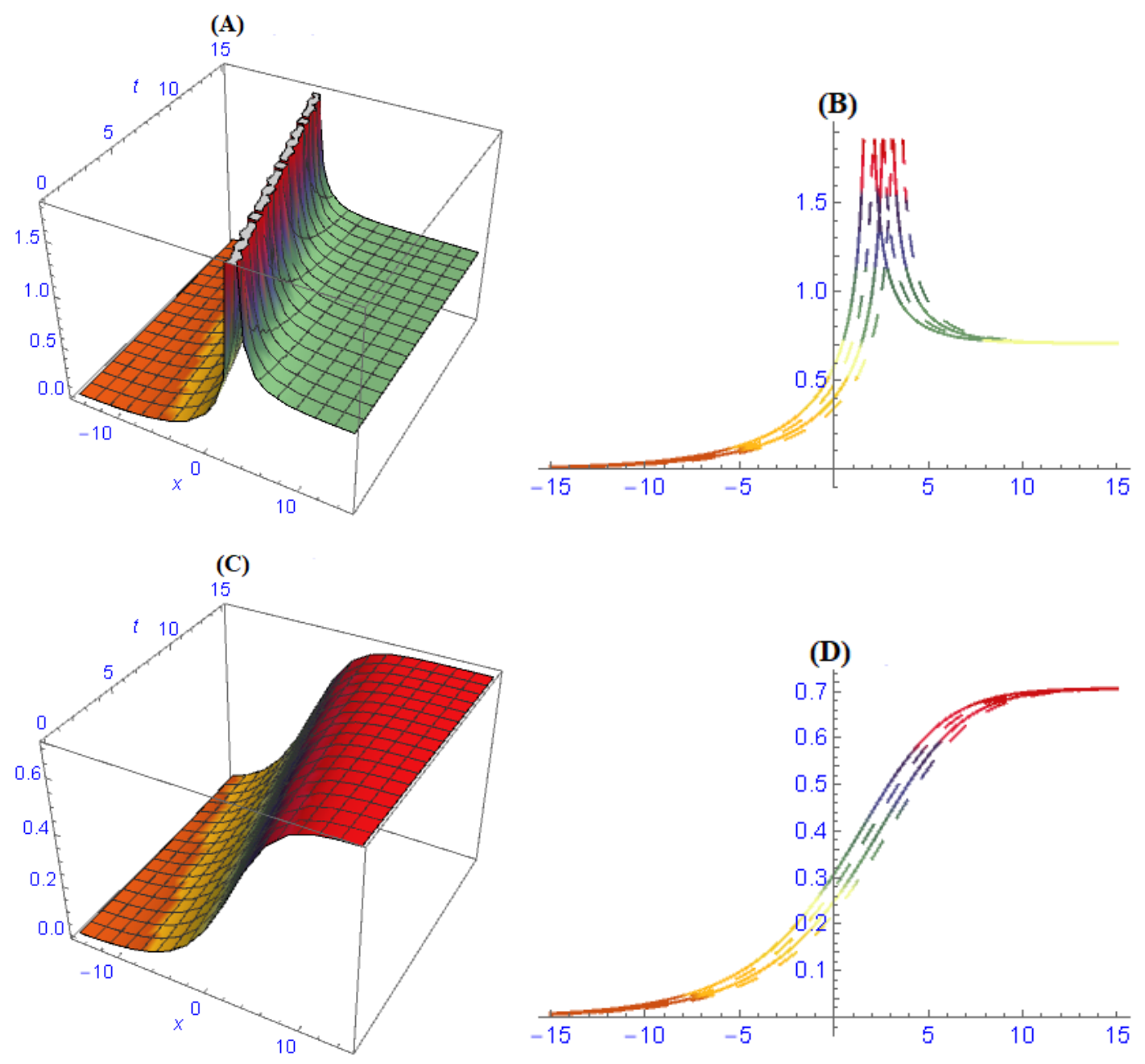

Figure 4. The graph of results (57) and (59) are depicted at dissimilar values of parameters and we obtained: (A) bright solitary wave and its 2-dim in (B), (C) anti-kink soliton and its 2-dim in (D).
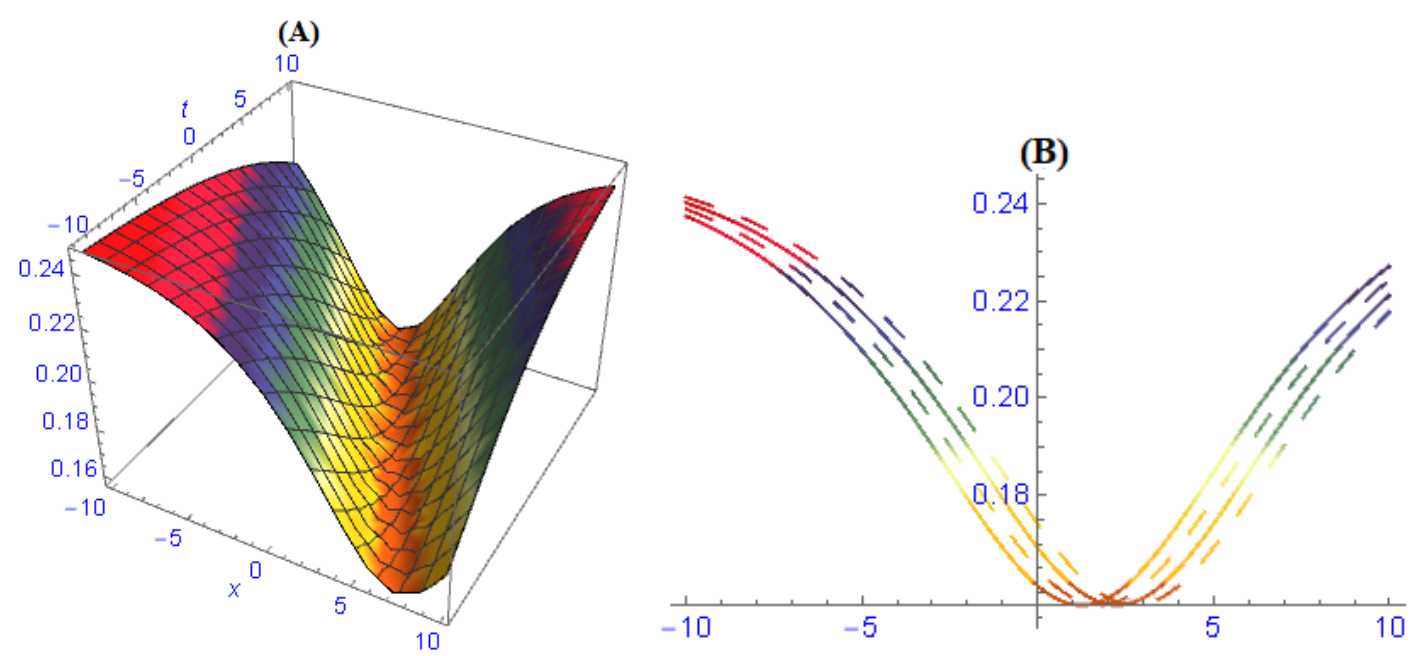

Figure 5. Cont. 

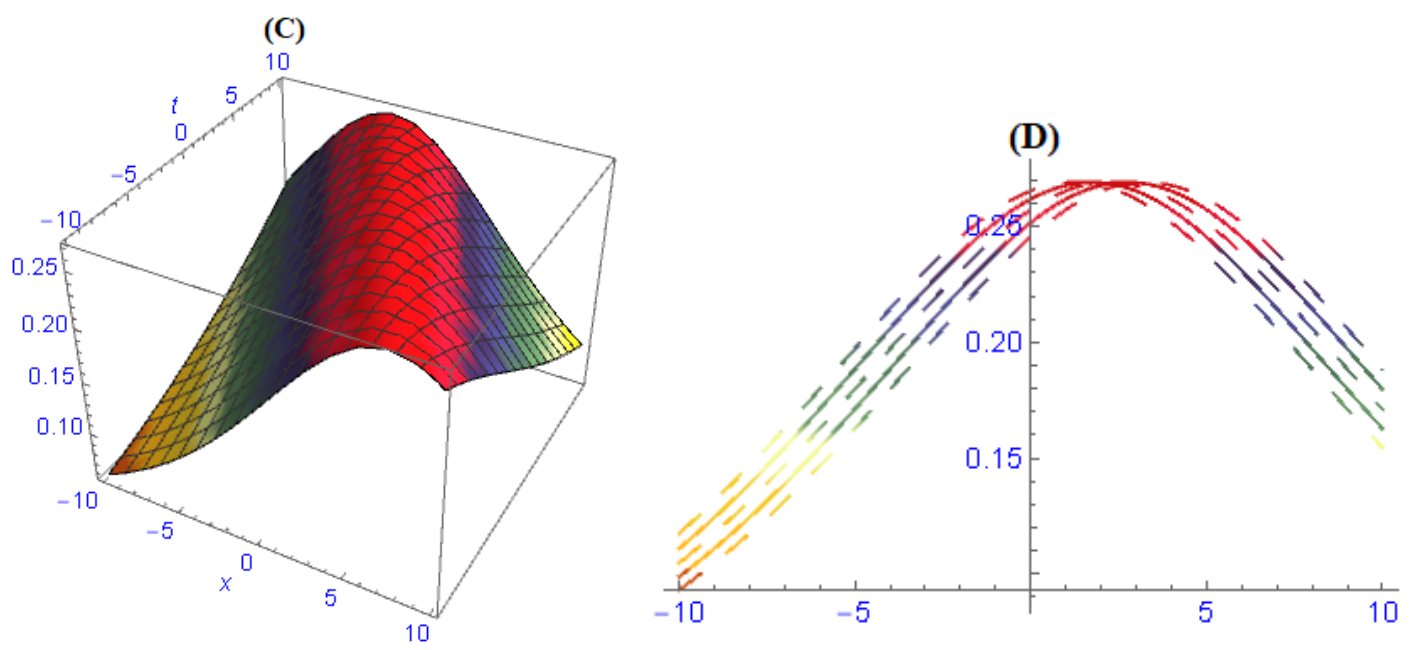

Figure 5. The structures of results (64) and (67) are depicted at dissimilar values of parameters: (A) dark compact type soliton and its 2-dim is in (B), (C), compact type soliton and its 2-dim is in (D).
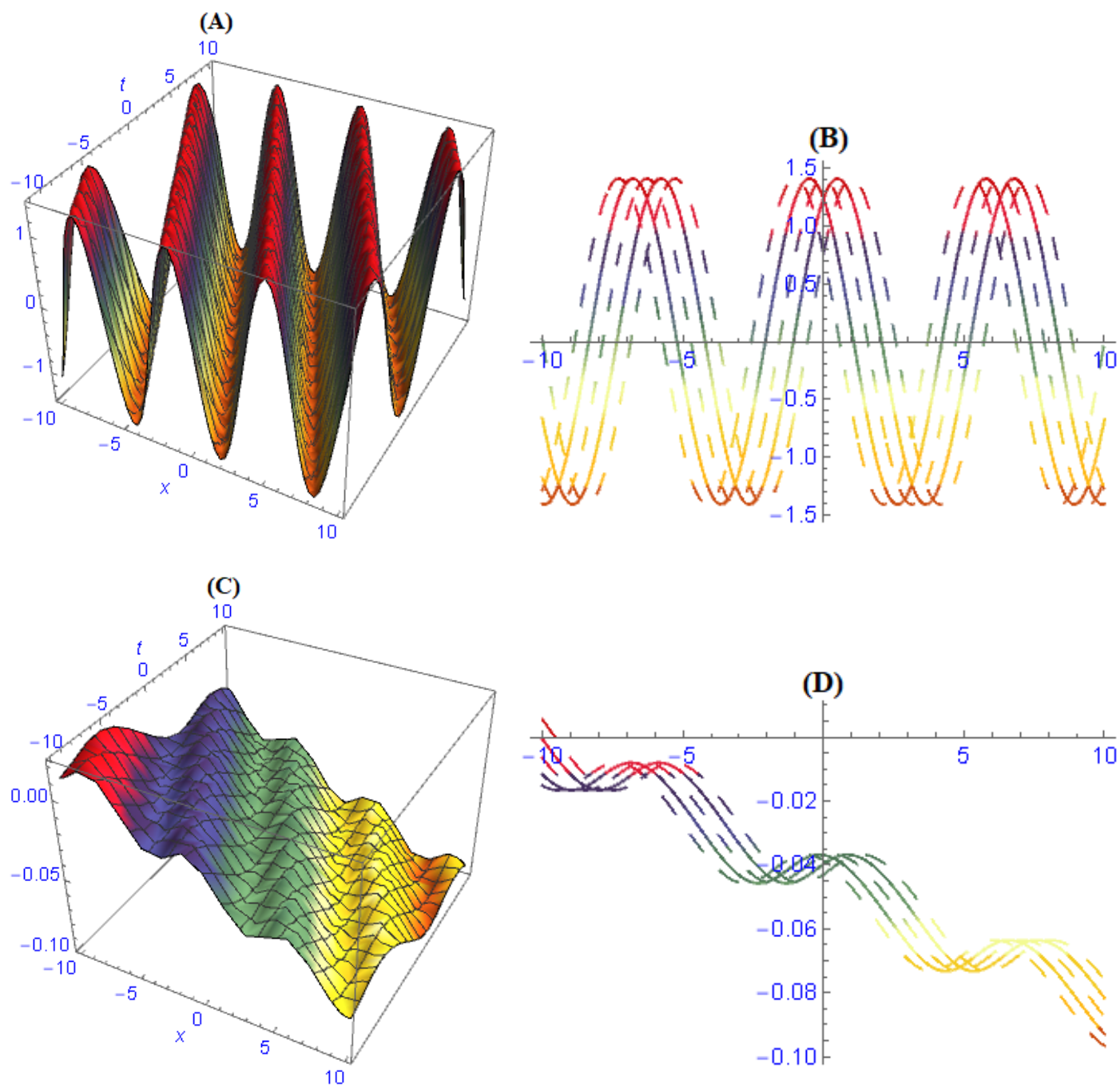

Figure 6. The structures of results (73) and (78) are depicted at dissimilar values of parameters: (A) periodic soliton and its 2-dim is in (B), (C) multi-kink type solitary wave and its 2-dim is in (D). 


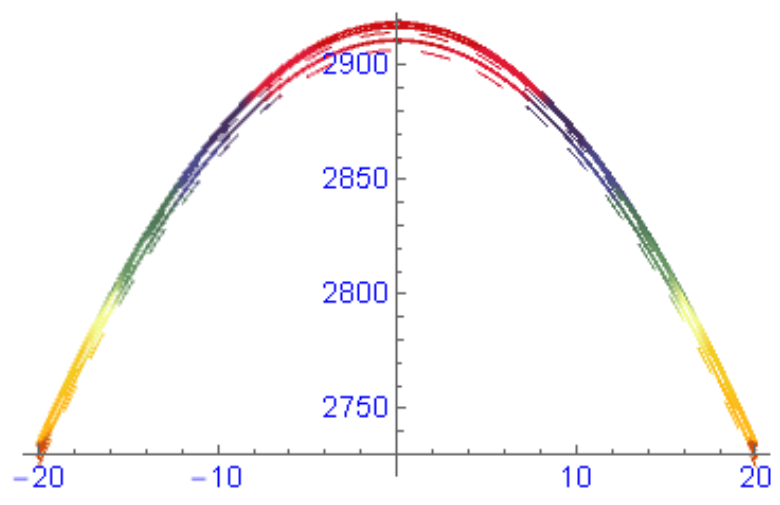

Figure 7. The graph of dispersion relation $k_{3}=k_{3}\left(k_{1}, k_{2}, \omega\right)$.

\section{Conclusions}

Three analytical methods have been successfully employed for $(3+1)$-dimension cubic-quintic complex Ginzburg-Landau equation and bright-dark solitons, breather-type and multi solitons interaction, kink and anti-kink solitons, and periodic and other solutions are constructed. The soliton profile is sensitive to entropy, i.e., due to the changes in the entropy amplitude and the width of solitons. The propagation of ultrashort optical solitons in optical fiber is modeled by this equation. The complex Ginzburg-Landau equation with broken phase symmetry has strict positive space-time entropy for an open set of parameter values. The motivation and purpose of this paper is to provide analytical methods to explore exact solutions which helps physicians, mathematicians, and engineers to understand the physical phenomena of this model. The obtained solutions of this article are very helpful in governing solitons dynamics. The traveling wave solutions can also be attained, which are constructed from existing techniques by giving special values to parameters involved in the methods. The model stability is examined by employing the modulation instability analysis, which shows that the model is stable. The computation work and constructed exact solutions endorse the easiness, effectiveness, and influence of the current techniques. These powerful techniques can be employed for several other nonlinear complex PDEs that are arising in mathematical physics.

Author Contributions: Conceptualization, D.L.; Data curation, X.Q.; Formal analysis, M.A.; Investigation, M.A.; Methodology, C.Y. and M.A.; Software, M.A.; Supervision, D.L.; Validation, N.N.; Writing—original draft, C.Y.; Writing-review \& editing, D.L. All authors have read and agreed to the published version of the manuscript.

Funding: This work was supported by the China Post-Doctoral Science Foundation, People's Republic of China (PRC) (Grant No. 2019M651715) and China Scholarship Council (CSC) (201908320301).

Conflicts of Interest: The authors declare no conflict of interest.

\section{References}

1. Lopez-Mariscal, C.; Gutierrez-Vega, J.C. In your phase: All about optical vortices. Opt. Photonics News 2009, 20, 10-13.

2. Desyatnikov, A.S.; Kivshar, Y.S.; Torner, L. Optical Vortices and Vortex Solitons. Prog. Opt. 2005, 47, $291-391$.

3. Agrawal, G.P. Nonlinear Fiber Optics, 5th ed.; Springer: Berlin/Heidelberg, Germany, 2013.

4. Nasreen, N.; Lu, D.; Arshad, M. Optical solitons of nonlinear Schrödinger equation with second order spatiotemporal dispersion and its modulation instability. Optik 2018, 161, 221-229. [CrossRef]

5. Arshad, M.; Seadawy, A.R.; Lu, D. Modulation stability and dispersive optical solitons solutions of higher order nonlinear Schrödinger equation and its applications in mono-mode optical fibers. Superlattices Microstruct. 2018, 113, 419-429. [CrossRef]

6. Khater, M.; Attia, R.A.; Lu, D. Explicit Lump Solitary Wave of Certain Interesting (3 + 1)-Dimensional Waves in Physics via Some Recent Traveling Wave Methods. Entropy 2019, 21, 397. [CrossRef] 
7. Aissat, A.; Mohammedi, T.; Alshehri, B. A geo-numerical approach for the classification of fixed points in the reduced model of the cubic-quintic complex Ginzburg-Landau equation. Optik 2015, 126, 5325-5330. [CrossRef]

8. Lu, D.; Yue, C.; Arshad, M. Traveling Wave Solutions of Space-Time Fractional Generalized Fifth-Order KdV Equation. Adv. Math. Phys. 2017, 2017, 6743276. [CrossRef]

9. Inc, M.; Bildic, N. Non-perturbative solution of the Ginzburg-Landau equation. Math. Comput. Appl. 2000, 5, 113-117.

10. Li, B.; Yang, C. Global well-posedness of the time-dependent Ginzburg-Landau superconductivity model in curved polyhedra. J. Math. Anal. Appl. 2017, 451, 102-116. [CrossRef]

11. Biswas, A. Temporal 1-soliton solution of the complex Ginzburg-Landau equation with power law nonlinearity. Prog. Electromagn. Res. 2009, 96, 1-7. [CrossRef]

12. Rougemont, J. Space-time invariant measures, entropy, and dimension for stochastic Ginzburg-Landau Equations. Commun. Math. Phys. 2002, 225, 423-448. [CrossRef]

13. LU, D.; Seadawy, A.R.; Arshad, M. Application of simple equation method on unstable nonlinear Schrödinger equations. Optik 2017, 140, 136-144. [CrossRef]

14. Succi, S. Lattice quantum mechanics: An application to Bose-Einstein condensation. Int. J. Mod. Phys. C 1998, 9, 1577-1585. [CrossRef]

15. Palpacelli, S.; Succi, S.; Spigler, R. Ground-state computation of Bose-Einstein condensates by an imaginary-time quantum lattice Boltzmann scheme. Phys. Rev. E 2007, 76, 036712. [CrossRef]

16. Palpacelli, S.; Succi, S. Quantum lattice Boltzmann simulation of expanding Bose-Einstein condensates in random potentials. Phys. Rev. E 2008, 77, 066708. [CrossRef]

17. Zhong, L.H.; Feng, S.D.; Dong, P.; Gao, S.T. Lattice Boltzmann schemes for the nonlinear Schrödinger equation. Phys. Rev. E 2006, 74, 036704. [CrossRef]

18. Akhmediev, N.N.; Afanasjev, V.V. Stability of the pulse like solutions of the quintic complex Ginzburg-Landau equation. J. Opt. Soc. Am. B 1996, 3, 1439-1449.

19. Cross, M.C.; Hohenberg, P.C. Pattern formation outside of equilibrium. Rev. Mod. Phys. 1993, 65, 851-1089. [CrossRef]

20. Aranson, I.S.; Kramer, L. The world of the complex Ginzburg-Landau equation. Rev. Mod. Phys. 2002, 74, 99-143. [CrossRef]

21. Gao, H.; Wang, X. On the global existence and small dispersion limit for a class of complex Ginzburg-Landau equations. Math. Meth. Appl. Sci. 2009, 32, 1396-1414. [CrossRef]

22. Marcq, P.; Chate, H.; Conte, R. Exact solutions of the one-dimensional, quintic complex Ginzburg-Landau equation. Physica D 1994, 73, 305-317. [CrossRef]

23. Charbonneau, J. Introduction to the Ginzburg-Landau Equations; V6T 1Z1; Department of Physics and Astronomy, University of British Columbia: Vancouver, BC, Canada, 2005.

24. Ortega, J.B.; Sardella, E.; Aguiar, J.A. Superconducting properties of a parallelepiped mesoscopic superconductor: A comparative study between the 2D and 3D Ginzburg-Landau models. Phys. Lett. A 2015, 379, 732-737. [CrossRef]

25. Siddheshwar, P.G. A series solution for the Ginzburg-Landau equation with a time-periodic coefficient. Appl. Math. 2010, 1, 542-554. [CrossRef]

26. Mihalache, D.; Mazilu, D.; Lederer, F.; Kartashov, Y.V.; Crasovan, L.C.; Torner, L.; Malomed, B.A. Stable vortex tori in the three-dimensional cubic-quintic Ginzburg-Landau equation. Phys. Rev. Lett. 2006, 97, 073904. [CrossRef] [PubMed]

27. Mihalache, D.; Mazilu, D.; Skarka, V.; Malomed, B.A.; Leblond, H.; Aleksic, N.B.; Lederde, F. Stable topological modes in two-dimensional Ginzburg-Landau models with trapping potentials. Phys. Rev. A 2010, 82, 023813. [CrossRef]

28. Skarka, V.; Aleksic, N.B.; Leblond, H.; Malomed, B.A.; Mihalache, D. Varieties of stable vertical solitons in Ginzburg-Landau media with radially inhomo-geneous losses. Phys. Rev. Lett. 2010, 105, 213901. [CrossRef]

29. Zhang, J.Y.; Yan, G.W. Lattice Boltzmann model for the complex Ginzburg-Landau equation. Phys. Rev. E 2010, 81, 066705. [CrossRef]

30. Zhang, J.Y.; Yan, G.W. Numerical studies based on higher-order accuracy lattice Boltzmann model for the complex Ginzburg-Landau equation. J. Sci. Comput. 2012, 52, 656-674. [CrossRef] 
31. Zhang, J.Y.; Yan, G.W. Three-dimensional lattice Boltzmann model for the complex Ginzburg-Landau equation. J. Sci. Comput. 2014, 60, 660-683. [CrossRef]

32. Fleischer, J.W.; Segev, M.; Efremidis, N.K.; Christodoulides, D.N. Observation of two-dimensional discrete solitons in optically induced nonlinear photonic lattices. Nature 2003, 422, 147-150. [CrossRef]

33. Benzi, R.; Succi, S.; Vergassola, M. The lattice Boltzmann equation: Theory and applications. Phys. Rep. 1992, 222, 145-197. [CrossRef]

34. Jeffery, K.; Pollack, R.; Rovelli, C. On the Statistical Mechanics of Life: Schrödinger Revisited. Entropy 2019, 21, 1211. [CrossRef]

35. Arshad, M.; Lu, D.; Rehman, M.-U.; Ahmed, I.; Sultan, A.M. Optical Solitary Wave and Elliptic function Solutions of Fokas-Lenells Equation in Presence of Perturbation Terms and its Modulation Instability. Phys. Scr. 2019, 94, 105202. [CrossRef]

36. Correa, F.; Martínez, C.; Troncoso, R. Hairy black hole entropy and the role of solitons in three dimensions. J. High Energy Phys. 2012, 2012, 136. [CrossRef]

37. Lu, D.; Seadawy, A.R.; Wang, J.; Arshad, M.; Farooq, U. Solitons Solutions of Generalized Third-Order Nonlinear Schrodinger Equation by two Mathematical Methods and its Stability. Pramana-J. Phys. 2019, 93, 44. [CrossRef]

38. Navickas, Z.; Bikulciene, L.; Rahula, M.; Ragulskis, M. Algebraic operator method for the construction of solitary solutions to nonlinear differential equations. Commun. Nonlinear Sci. Numer. Simul. 2013, 18, 1374-1389. [CrossRef]

39. Arshad, M.; Seadawy, A.R.; Lu, D.; Wang, J. Travelling wave solutions of Drinfel'd-Sokolov-Wilson, Whitham-Broer-Kaup and (2+1)-dimensional Broer-Kaup-Kupershmit equations and their applications. Chin. J. Phys. 2017, 140, 136-144. [CrossRef]

40. Arnous, A.H.; Seadawy, A.R.; Alqahtani, R.T.; Biswas, A. Optical solitons with complex Ginzburg-Landau equation by modified simple equation method. Optik 2017, 55, 780-797. [CrossRef]

41. Seadawy, A.R.; Arshad, M.; Lu, D. Stability analysis of new exact traveling-wave solutions of new coupled KdV and new coupled Zakharov-Kuznetsov systems. Eur. Phys. J. Plus 2017, 132, 162. [CrossRef]

42. Zhang, J.; Yan, G.; Wang, M. Lattice Boltzmann simulations for the vortex tori pattern in the three-dimensional cubic-quintic complex Ginzburg-Landau equation. J. Comput. Phys. 2016, 306, 311-319. [CrossRef]

43. Inc, M.; Aliyu, A.I.; Yusu, A.; Baleanu, D. Optical Solitons and Modulation Instability Analysis with (3 + 1)-Dimensional Nonlinear Shrödinger Equation. Superlattices Microstruct. 2017, 112, 296-302. [CrossRef]

(C) 2020 by the authors. Licensee MDPI, Basel, Switzerland. This article is an open access article distributed under the terms and conditions of the Creative Commons Attribution (CC BY) license (http:/ / creativecommons.org/licenses/by/4.0/). 